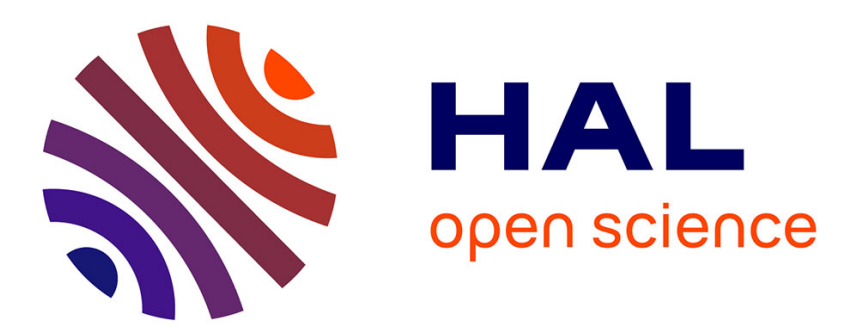

\title{
Image processing using proper orthogonal and dynamic mode decompositions for the study of cavitation developing on a NACA0015 foil
}

\author{
Sébastien Prothin, Jean-Yves Billard, Henda Djeridi
}

\section{- To cite this version:}

Sébastien Prothin, Jean-Yves Billard, Henda Djeridi. Image processing using proper orthogonal and dynamic mode decompositions for the study of cavitation developing on a NACA0015 foil. Experiments in Fluids, 2016, 57 (10), pp.157-182. 10.1007/s00348-016-2246-1 . hal-01377045

\section{HAL Id: hal-01377045 \\ https://hal.science/hal-01377045}

Submitted on 6 Oct 2016

HAL is a multi-disciplinary open access archive for the deposit and dissemination of scientific research documents, whether they are published or not. The documents may come from teaching and research institutions in France or abroad, or from public or private research centers.
L'archive ouverte pluridisciplinaire HAL, est destinée au dépôt et à la diffusion de documents scientifiques de niveau recherche, publiés ou non, émanant des établissements d'enseignement et de recherche français ou étrangers, des laboratoires publics ou privés. 


\title{
Image processing using proper orthogonal and dynamic mode decompositions for the study of cavitation developing on a NACA0015 foil
}

\author{
Sebastien Prothin ${ }^{1} \cdot$ Jean-Yves Billard $^{2} \cdot$ Henda Djeridi $^{3}$
}

\begin{abstract}
The purpose of the present study is to get a better understanding of the hydrodynamic instabilities of sheet cavities which develop along solid walls. The main objective is to highlight the spatial and temporal behavior of such a cavity when it develops on a NACA0015 foil at high Reynolds number. Experimental results show a quasisteady, periodic, bifurcation domain, with aperiodic cavity behavior corresponding to $\sigma / 2 \alpha$ values of $5.75,5,4.3$ and 3.58. Robust mathematical methods of signal postprocessing (proper orthogonal decomposition and dynamic mode decomposition) were applied in order to emphasize the spatio-temporal nature of the flow. These new techniques put in evidence the 3D effects due to the reentrant jet instabilities or due to propagating shock wave mechanism at the origin of the shedding process of the cavitation cloud.
\end{abstract}

\section{List of symbols}

$\begin{array}{ll}B & \text { Span }(\mathrm{m}) \\ C & \text { Chord }(\mathrm{m}) \\ C_{n}^{(n)} & \text { Convergence matrix } \\ l_{\mathrm{c}} & \text { Cavity length }(\mathrm{m})\end{array}$

Henda Djeridi

henda.djeridi@legi.grenoble-inp.fr

1 Département Aérodynamique, Energie et Propulsion, Institut Supérieur de l'Aéronautique et de l'Espace, (ISAE Supaéro), BP 54032, 31055 Toulouse Cedex 04, France

2 Département Mécanique et Energétique en Environnement Naval, CC 400, Institut de Recherche de l'Ecole Navale (IRENav EA 3634), 29240 Brest Cedex 9, France

3 Laboratoire des Ecoulements Géophysiques et Industriels (LEGI, UMR 5519), Domaine Universitaire, BP 53, 38041 Grenoble Cedex 9, France

$\begin{array}{ll}P_{\text {ref }} & \text { Reference pressure }(\mathrm{Pa}) \\ P_{\mathrm{v}} & \text { Vapor pressure }(\mathrm{Pa}) \\ V_{\text {ref }} & \text { Reference velocity }\left(\mathrm{m} \mathrm{s}^{-1}\right) \\ \sigma & \text { Cavitation parameter } \\ \rho & \text { Fluid density }\left(\mathrm{kg} \mathrm{m}^{-3}\right) \\ \alpha & \text { Incidence }\left({ }^{\circ}\right) \\ \Phi & \text { Orthogonal function (POD) } \\ \varphi & \text { Phase angle }\left(^{\circ}\right) \\ R e & \text { Reynolds number } \\ N_{\mathrm{T}} & \text { Number of snapshots/images } \\ N_{\mathrm{S}} & \text { Number of samples } \\ N_{\text {modes }} & \text { Number of POD modes } \\ a_{i} & \text { POD temporal coefficient } \\ X_{i} & \text { POD spatial mode } \\ \lambda_{i} & \text { Eigenvalues of POD or DMD } \\ f_{\mathrm{s}} & \text { Sample frequency } \\ \Psi_{i} & \text { DMD Mode }\end{array}$

\section{Introduction}

Cavitation on hydrofoils has been widely investigated (Le et al. 1993; Furness and Hutton 1975; Stutz and Reboud 1997: Pham et al. 1999; Laberteaux and Ceccio 2001 for example). The characteristics of the pressure field govern the type of cavitation which appears. For low pressure gradients (corresponding to the low values of incidence angle of the foil), transient bubbles have been observed on the suction side near the leading edge of the foil. For strong pressure gradients, sheet cavitation has been observed at the same location. Sheet cavitation is frequently observed on wearing surfaces and is strongly unsteady at the closure part of the pocket; furthermore, depending on the parameters of the flow (incidence, cavitation number, Reynolds numbers), several types of sheet cavitation has 
been obtained (stable, unstable, self-oscillating, etc.). In most cases, cavitation is an undesirable phenomenon, responsible for vibrations, noise, reduced flow rates, loss of performance, large pressure fluctuations and damage to solid structures. Considerable effort has been made to understand the physical phenomena of both sheet and cloud cavitation, and numerous studies have dealt with the different parameters which govern the shape and behavior of the cavity. This kind of cavitation has an unsteady behavior shedding vapor structures in the flow. The shedding mechanism comes either from a reentrant jet structure that cuts the cavity or from an interfacial wave the amplitude of which may lead to the separation of a vapor cloud. Previous research concerning sheet unsteadiness as a function of the parameter $\sigma / 2 \alpha$, where $\sigma$ and $\alpha$ denote the cavitation number and the incidence angle of the foil, respectively, can be quoted (Chahine and Hsiao 2000: Kawakami et al. 1997, 2008; Arndt et al. 2000; Kjeldsen and Arndt 2001; Fujii et al. 2007). For unsteady cavities $\left(l_{\mathrm{c}} / C>0.5\right)$, Arndt et al. (2000) pointed out a modification of the sheet dynamics for $\sigma / 2 \alpha=4$ on a NACA0015 profile correlated with a modification of the shedding frequency. For example, for the 2D NACA foil, periodic cloud shedding was observed with oscillation frequencies resulting in a parameter $\sigma / 2 \alpha<4$ or $>4$. A bifurcation of shedding mechanism around the value of $\sigma / 2 \alpha=4$ for different configurations has been observed by several authors. This shedding mechanism has been explored in detail by Ganesh et al. (2014) in wedge geometry. Indeed, based on the measured void ratio using X-ray densitometry and the measured shock velocity, the pressure before and after the shock can be predicted (Ganesh et al. 2014). This behavior was characterized by three shedding modes where both bubbly flow shock wave and reentrant jet phenomena can occur. This complex form of sheet and cloud cavitation is associated with two- and three-dimensional vapor phase structures though it can be difficult to distinguish between the temporal and spatial scales responsible for the vapor phase dynamics. Even if the parameter that governs the transition mechanism seems correctly defined, work must be performed concerning the variations of the shedding frequency and the spatial dynamics of the cavity. Based on the work of Prothin et al. (2012), Danlos et al. (2014) used the proper orthogonal decomposition (POD) method, applied on acquired images, to detect a cavitation regime in a grooved convergent-divergent nozzle. They studied the effects of the presence of the grooved plate on the dynamics of the sheet cavity. As observed by Prothin et al. (2012), POD is an efficient tool to detect the shedding process and to discriminate stable or unstable states of the vapor phase. Nevertheless, in cavitation studies focused on partial cavity behavior, the cavity is frequently assumed to remain two-dimensional. This assumption is only based on the fact that the spanwise velocity component of the sub-cavitating basic flow can be neglected as compared to the chordwise component. This assumption has been verified by 3D flow measurements where, even for large incidence angles of the foil, the spanwise velocity component remains very small compared to the other components (Prothin et al. 2013). In previous work on cavitating convergent-divergent nozzle, only side views of sheet cavities were acquired and analyzed as a two-dimensional entity. This is important when considering three-dimensional effects due to the reentrant jet instabilities or due to a propagating shock wave mechanism at the origin of shedding process of the cavitation cloud. Three-dimensional effects lead to the presence of a non-commensurable additional frequency in the shedding process.

For this reason, the present paper introduces a spacetime study of a sheet cavity developing on a NACA0015 two-dimensional profile making use of two mathematical tools: POD (Sirovich 1987) and dynamical mode decomposition (DMD) (Schmid 2010) applied to space-time signals generated by high-speed image sequences. Schmid (2010) introduced the DMD method to extract information from the dynamic mode based on Koopman's analysis of dynamic systems (Rowley et al. 2009). The extracted modes can be interpreted as generalizations of global mode stability and are used to describe the physical mechanism with Fourier modes corresponding to spatial structures of the sheet cavity. This complementary approach allows us to demonstrate the spectral contribution of the longitudinal and spanwise vapor structures of each cycle. Moreover, even if the main structures remain two-dimensional the sheet behavior may become three dimensional as shown by De Lange and de Bruin (1998). This aspect will be addressed below.

For the present purpose, four configurations have been studied, $\sigma / 2 \alpha=5.73,5.01,4.30$ and 3.58. The first value corresponds to a low-amplitude harmonic behavior, the second to large-amplitude behavior, the third is near to transition and the last is aperiodic. Two different methods have been used or developed to characterize the spatial dynamics of the cavity and its 2D/3D behavior. Concerning the first method, from top visualizations and span averaging of the gray levels, the temporal evolution of the sheet cavity is constructed and analyzed to describe the frequency behavior and the convective velocity of vapor structures. The second method, using the POD and DMD, focuses on the most energetic shedding modes and their 2D/3D behavior 
allowing a characterization of the dynamic bifurcations of cavitation behavior.

\section{Experimental setup}

\subsection{Experimental facility and flow description}

Experiments were conducted in the cavitation tunnel of the French naval academy (CTNA). The CTNA is fitted with a 1 -m-long square test section of $196 \times 196 \mathrm{~mm}^{2}$. The test section is made of altuglas which allows visualization of the profile through three of the four sides of the vein. The fluid (fresh water) flow can be set between 3 and $15 \mathrm{~ms}^{-1}$ and the pressure between 30 mbar and 3 bar in the test section. For the present investigation, a 2D NACA0015 profile fixed at mid-height was used (Chord $C=100 \mathrm{~mm}$, Span $B=192 \mathrm{~mm}$ ). To avoid light reflections on the stainless iron foil surface, it was coated with a single layer of matt black paint. Four different conditions where sheet cavity develops were investigated. They differ in the value of the cavitation index, $\sigma$, defined as:

$\sigma=\frac{P_{\text {ref }}-p_{\mathrm{v}}}{\frac{1}{2} \rho V_{\text {ref }}^{2}}$

where $P_{\text {ref }}$ and $V_{\text {ref }}$ are the pressure and the flow velocity in the reference section, respectively, and $p_{\mathrm{v}}$ is the vapor pressure at the experiment temperature. In the present case, the reference velocity has been set to $5 \mathrm{~m} / \mathrm{s}$ and the incidence angle of the foil to $10^{\circ}$. The cavitation index of 1.25 , 1.75 and 2 (corresponding to $\sigma / 2 \alpha=3.58,4.3,5,5.75$ ) was fixed via a reference pressure modification. Taking into account that dissolved gas concentration inside the water plays a major role in cavitation inception (Kawakami et al. 2008), the main objective was to obtain a reproducible cavitating conditions. To achieve reproducible experiments, a degasification protocol was established during $1 \mathrm{~h}$ for each condition and the dissolved $\mathrm{O}_{2}$ concentration has been measured (acquired with an Orbisphere $\mathrm{MOCA} \mathrm{O}_{2}$ probe) at the beginning and during all experiments. The level of dissolved air is constant, fixed at $3.5 \mathrm{ppm}$, for each test condition, and the sensor precision was $\pm 1 \%$. After $1 \mathrm{~h}$, the rms value of the concentration was about $8.29 \times 10^{-3}$ and the relative deviation of $0.25 \%$.

The measurement of the water's temperature was required in order to calculate the vapor pressure and to set the selected cavitation number. The recorded water temperature varied within the range of $18-21^{\circ} \mathrm{C}$ depending on operating and atmospheric conditions. The mean temperature was $19^{\circ}$ for all tests. All operating points have been fixed by the upstream pressure using an absolute pressure sensor with an uncertainty of about $300 \mathrm{~Pa}$, and the sigma value was given with a precision of 0.02 . Taking into account the regulation system, upstream velocity was given with an uncertainty of $0.02 \mathrm{~m} / \mathrm{s}$ and the monitoring control of incidence angle gave $\pm 7^{\prime}$.

\subsection{Description of data sets}

Pictures have been taken using a Photron FASTCAM SA3 model 120K-M2 fast video camera positioned above the test section to observe the suction side of the foil where the cavity develops. The image rate was set to $2000 \mathrm{fps}$ to follow precisely the cavity behavior. Each set of data contains $N_{\mathrm{T}}=5000$ images and represents $2.5 \mathrm{~s}$ of observation. Each rough image comprises $512 \times 256$ pixels, and thus, the spatial resolution is $\mathrm{d} x_{\text {pix }}=U * \mathrm{~d} T / G$ with $G=0.2 \mathrm{~mm} /$ pix. The high-speed observations were illuminated with two continuous cold lights positioned at $45^{\circ}$ upstream and downstream the foil (Fig. 1). The camera was placed at the vertical position of the foil. Clouds are not shadowed by other structures and the black part corresponds to the liquid phase. The exposure time of $0.5 \mathrm{~ms}$, the shutter time of $20 \mu$ s and a depth of field of $10 \mathrm{~cm}$ are chosen to avoid any blur or saturation of the recorded images as shown in Fig. 2.

\subsection{Image processing}

The rough data set contains pictures of the sheet cavity, one of which is presented in Fig. 3. Two different methods of image processing have been applied to each data set. The

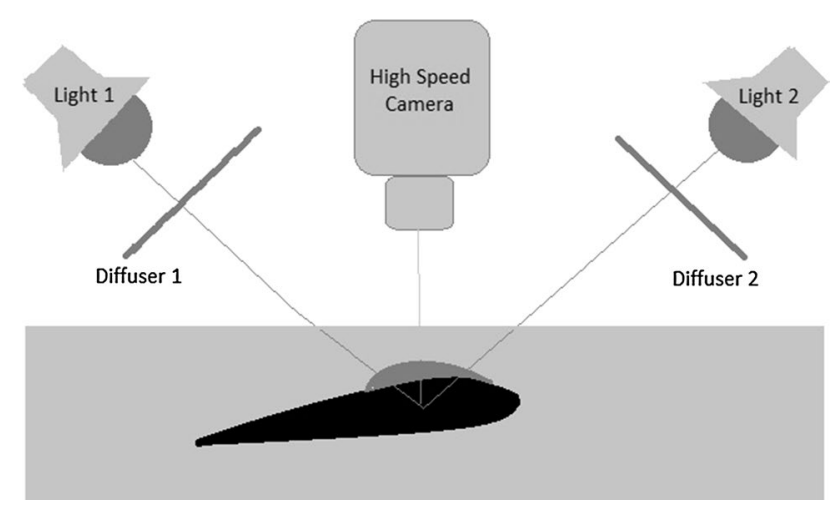

Fig. 1 Scheme of lighting of the foil 
Fig. 2 Gray scale histogram of a typical image

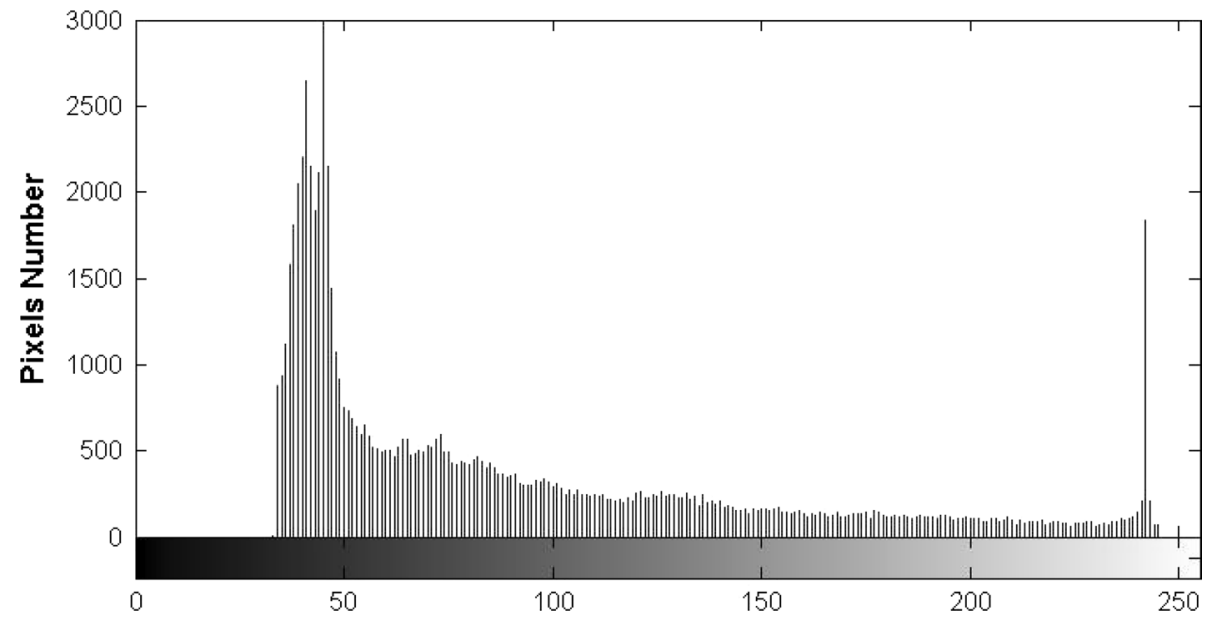

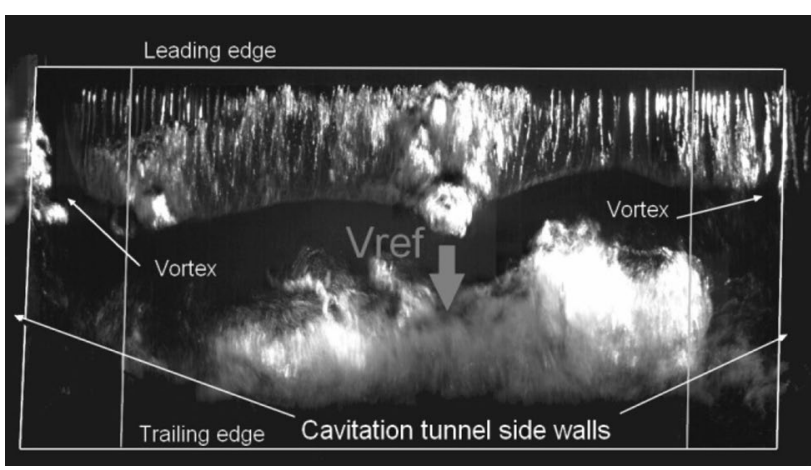

Fig. 3 Unsteady sheet cavity on NACA0015 2D profile $(\sigma=1.75$, $\left.\alpha=10^{\circ}\right)$

first method makes the classical assumption of 2D behavior of the cavity and is described precisely in the next paragraph. The second is an application of the POD applied to the gray levels of each image. This method can thus describe both the mean extend of the sheet cavity with its temporal behavior and the spanwise variations of the cavity which can be significant when the cavitation index is decreased. The preprocess is common for the two methods:

- The first step of the preprocess consists in an unwarping of the picture. During this step, the trapezoidal area surrounded by a white frame is projected on a rectangular area whose aspect ratio is equal to the foil aspect ratio.

- In the second step, the two cavitating vortices which can be seen on both side walls are eliminated and the central area of the picture, where a mean two-dimensional behavior is expected, is retained for following steps. This image is formed by $N_{\mathrm{s}}=75,000$ pixels ( 250 rows $\times 300$ columns). Each pixel is represented by a gray value between 0 and 255 . The resulting picture is presented in Fig. 4.
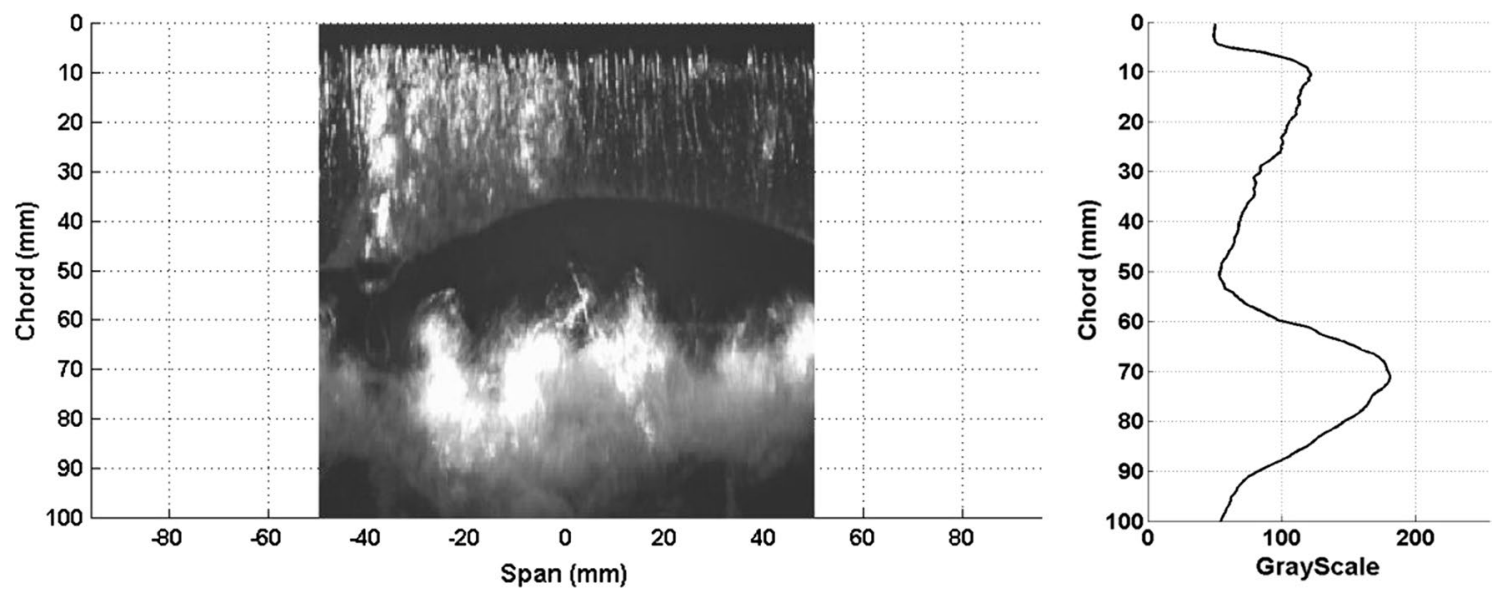

Fig. 4 Preprocessed image and mean gray profile 

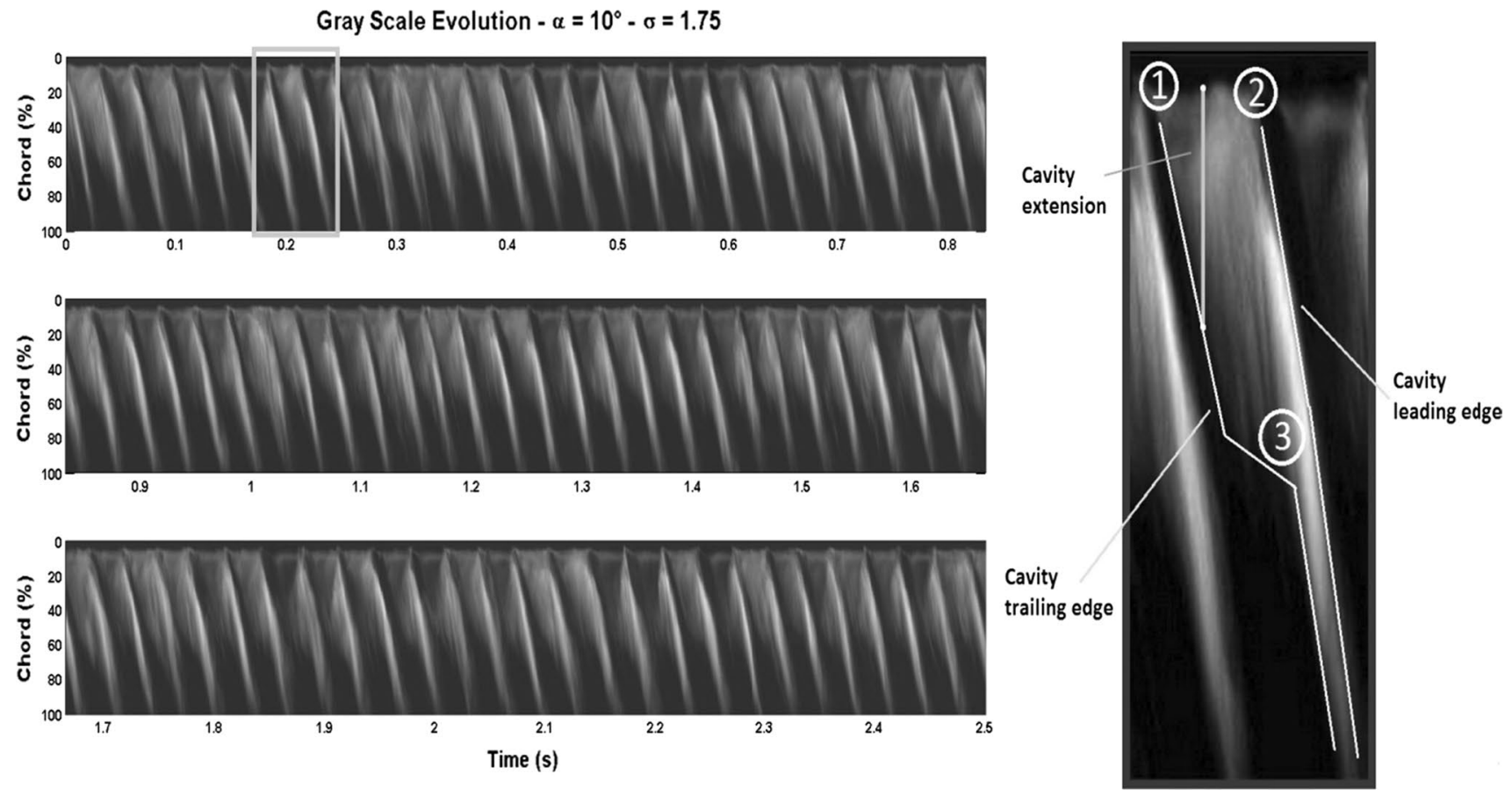

Fig. 5 Space-time diagram characteristic of the time dependence of the cavity (mean evolution of cavity extent with time)

\subsubsection{Method 1: 2D averaging in the spanwise direction}

A mean evolution of gray levels over the chord length is evaluated through the spatial averaging of all the columns of pixels over the span of the profile. Thus, each image is represented by a single column of pixels characteristic of the sheet cavity at each moment when the picture has been taken. The pixel values of this column are represented in Fig. 4 which can be described as the mean profile of the image. The next step of this process consists in the aggregation of these columns in a space-time diagram. The resulting image, shown in Fig. 4 ( $N_{\mathrm{T}}$ columns $\times 250$ rows), is representative of the cavity behavior during the $2.5 \mathrm{~s}$ of film duration. The time-dependent variability of the cavity extent is clearly observable. Furthermore, the periodic behavior of the cavity is attested by the repeatability of the same pattern over the abscissa axis.

From the image in Fig. 5, the cavity behavior can be described. The cavity appears close to the leading edge (1) and increases up to point (2) where the reentrant jet cuts off the cavity a little downstream of the leading edge. Immediately, the next pattern appears and will follow the same evolution. Just after the detachment of the cavity, the convective velocity of the structure on the trailing edge is modified (3) and due to the different pressure value between the leading and trailing edge, the cavity collapses. The period of the phenomenon is represented by the time interval between points (1) and (2). Moreover, as a function of the cavitation number, different behaviors are observed. The mean length of the cavity has been determined using the procedure proposed by Dular (2004) (Danlos et al. 2014) based on the standard deviation of the gray scale maps. This procedure has been applied both for the opening and closure of the cavity. The position of the closure of the cavity is evaluated with top view-acquired images. The mean and standard deviation of the gray levels are calculated (Fig. 6). Near the leading edge, gray levels oscillate between 0 value (liquid phase) and 250 (vapor phase), and when the maximum value is detected the cavity is established. The decrease in the rms value corresponds to the oscillations of the closure of the cavity; the cavity length is then evaluated. For the lower $\sigma$ value, the curve presents a plateau value of the standard deviation; the end of the plateau has been retained to define the closure position.

\subsubsection{Method 2: POD of the plan form image of the cavity}

The POD method introduced by Lumley (1970) leads to an energetic ${ }^{1}$ decomposition and gives a deterministic set of orthogonal functions called $\phi^{n}(X)$ and solution of a Fredholm equation. $N\left(X_{m}, t_{k}\right)$ is the matrix representation of the process, $m$ and $k$ being, respectively, the row and column indices. The column number of $N$ is equal to $N_{\mathrm{T}}$, and the

\footnotetext{
$\overline{1}$ In the POD meaning the energy of a totally black image is equal to zero, and the one of a totally white image reaches its maximum.
} 
Fig. 6 Standard deviation of the gray scale maps versus chord position

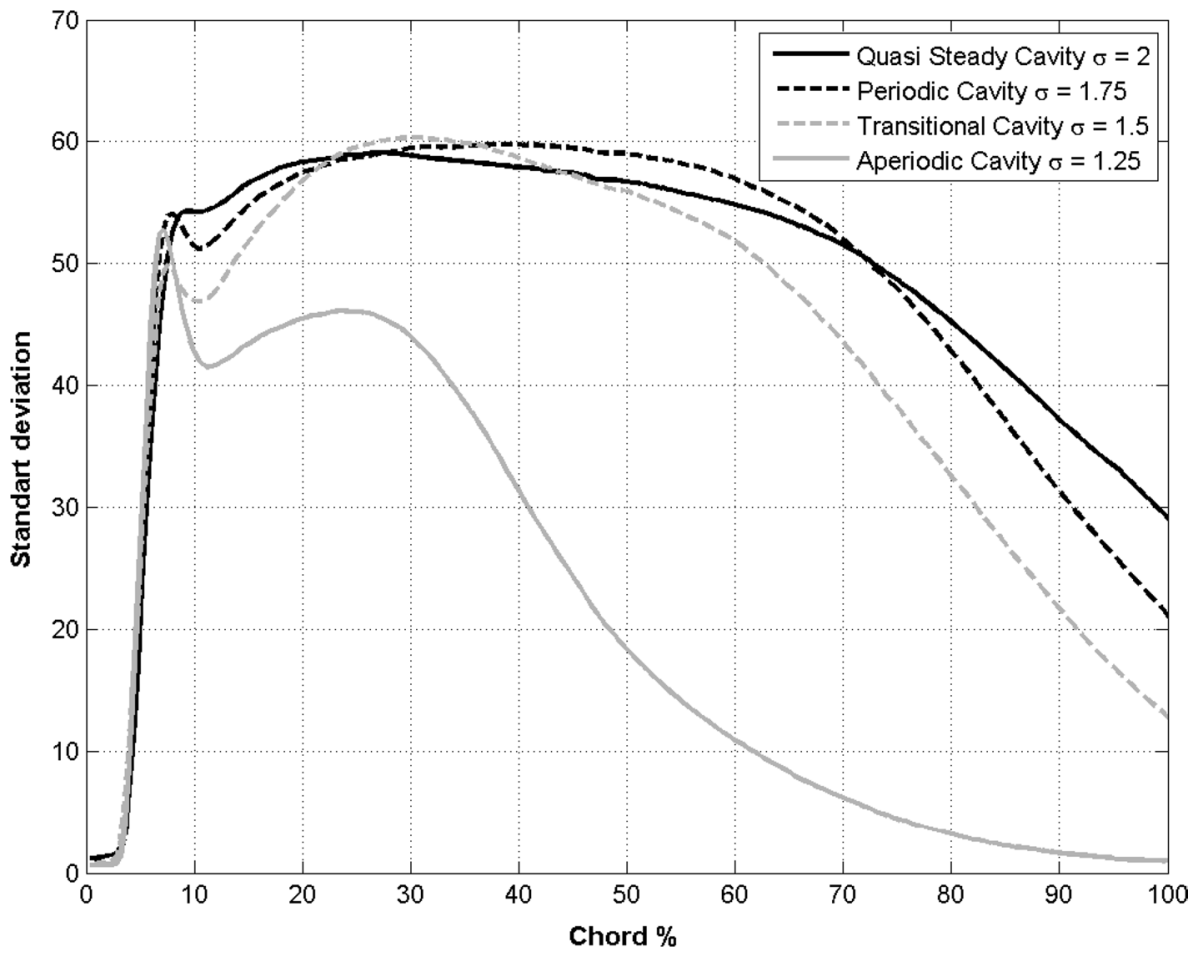

row number is equal to $N_{\mathrm{s}}=250 \times 300$ (each value in the column corresponds to one gray level of an image pixel).

$\sum_{l=1}^{N_{\mathrm{S}}} \sum_{r=1}^{N_{\mathrm{T}}} E\left[N_{i}\left(X_{p}, t_{q}\right) N_{j}^{*}\left(X_{l}, t_{r}\right)\right] \Phi_{j}^{(n)}\left(X_{l}, t_{r}\right) \Delta X=\lambda^{(n)} \Phi_{i}^{(n)}\left(X_{p}, t_{q}\right)$

where $E\left[N_{i}\left(X_{p}, t_{q}\right) N_{j}^{*}\left(X_{l}, t_{r}\right)\right]$ is the space-time tensor of two-point correlation, $E[\cdot]$ is the set average and $\phi(n)$ corresponds to the $n$th eigenvector associated with the eigenvalue $\lambda^{(n)}$. This value is representative of the "energy" content of the $n$th mode. Assuming that $N$ is an ergodic process, its mean can be estimated by a temporal averaging over all the time samples and thus eigenvectors are only space dependent.

$\sum_{l=1}^{N_{\mathrm{S}}} R_{i j}\left(X_{p}, X_{l}\right) \Phi_{j}^{(n)}\left(X_{l}\right) \Delta X=\lambda^{(n)} \Phi_{i}^{(n)}\left(X_{p}\right)$

where

$R_{i j}\left(X_{p}, X_{l}\right)=\overline{N_{i}\left(X_{p}\right) N_{j}\left(X_{l}\right)}=\frac{1}{N_{\mathrm{T}}} \sum_{q=1}^{N_{\mathrm{T}}} N_{i}\left(X_{p}, t_{q}\right) N_{j}\left(X_{l}, t_{q}\right)$

is the time average space correlation tensor. The $\phi(n)(X)$ are spatially orthogonal.
$\Phi_{i}^{(n)}\left(X_{\mathrm{P}}\right) \cdot \Phi_{i}^{(m)}\left(X_{\mathrm{P}}\right)=\delta_{n m}$

where \langle\rangle signifies the spatial average operator.

The projection of an image on the $n$th POD mode gives the temporal random coefficient $a^{(n)}\left(t_{q}\right)$. Each image is thus represented by a linear combination of the $N_{\mathrm{T}}$ modes weighted by its corresponding random coefficient:

$N_{i}\left(X_{p}, t_{q}\right)=\sum_{n=1}^{N_{\mathrm{T}}} a^{(n)}\left(t_{q}\right) \Phi_{i}^{(n)}\left(X_{p}\right)$

This method has been formalized by Sirovich (1987) and is referred to as the snapshot method. The decreasing sort of the eigenvalues reveals the most dominant spatial modes. Thus, the reconstruction (1) can be extended to the most energetic modes $\left(N_{\text {modes }}<N_{\mathrm{T}}\right)$ representative of the process. Each snapshot can thus be reconstructed using part of the modes. Particularly, when the process is dominated by a periodic phenomenon, as in the present study, the first POD modes are organized in pairs, both representative of the same orthogonal component of the periodic process (Ma and Karniadakis 2002). A statistical estimation, evaluated using a given number of samples, is considered converged if an increase in the number of samples leads to the same estimation. For this study, the reference has been chosen on the POD carried out on $N_{\mathrm{T}}$ samples. In the present case, $n_{\text {samples }}$ were $250,500 \ldots 4750$. 
Hekmati et al. (2011) proposed conducting this analysis with the computation of the following correlation coefficient $C_{n_{\text {samples }}^{(n)}}^{(\text {: }}$

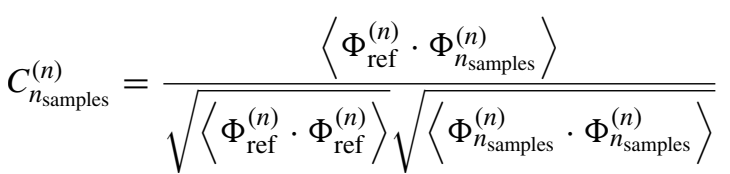

where $n$ is the mode number. Thus, $\Phi_{\text {ref }}^{(n)}$ corresponds to the $n$th mode computed with $N_{\mathrm{T}}$ samples and $\Phi_{n_{\text {samples }}}^{(n)}$ to the same mode evaluated with $n_{\text {samples }}$ snapshots. $C_{n_{\text {samples }}^{(n)}}$ is equal to 1 if the $\Phi_{n_{\text {samples }}}^{(n)}$ is identical to $\Phi_{\text {ref }}^{(n)}$ and decreases when differences appears in the two evaluations.

\subsubsection{Method 3: DMD of the plan form image of the cavity}

Schmid (2010) introduced a DMD to extract information from a Koopman flow field based on the analysis of such a system (Rowley et al. 2009). The extracted modes that can be interpreted as a generalization of modes of overall stability can also be used to describe the underlying physical mechanism that is captured in the data sequence. The relevance of the present work is to compare the classical POD with DMD, as this not only helps to retrieve relevant spatial structures (cyclic sheet cloud convected along the profile), but also to extract the corresponding Fourier modes. This additional information allows identifying the spectral contribution of longitudinal and transverse steam structures. The DMD method assumes there is an operator of evolution A of the dynamic acting on a set of observable $N(t)$ :

$N_{i+1}=A N_{i}$

The method returns to decompose the operator, seeking its own modes $\Psi_{i}$ and eigenvalues $\lambda_{i}$. By having this information available and assuming that spectrum step is sufficiently rich and therefore the associated eigenfunctions form a basis of the space phase, one can easily return to the equation:

$N_{i}=A^{i} N_{0}$

$N_{i}=\sum_{k}^{N t} v_{k 0} \lambda_{k}^{i} \psi_{k}$

where $v_{k 0}$ are the projection coefficients of the first field on a modal basis. $\Psi_{i}$ and $\lambda_{i}$ are estimated by decomposing a similar matrix to the evolution operator A. It is assumed that:

$N_{\mathrm{NT}}=c_{0} N_{0}+c_{1} N_{1}+\cdots+c_{N-1} N_{N-1}$

using $K_{0}^{N-1}$ the Krylov space $\left\{N_{0}, N_{1}, \ldots, N_{N-1}\right\}$.
$A K_{0}^{N-1}=K_{1}^{N} C+r, \quad$ with $C=\left[\begin{array}{ccc}0 & \cdots & c_{0} \\ 1 & \ddots & c_{1} \\ 0 & \ddots & c_{2} \\ \vdots & \ddots & \vdots \\ 0 & 0 & c_{N-1}\end{array}\right]$

$C$ is, in the case of zero residue, a matrix similar to the operator $A$, and yet of a compact size.

The DMD algorithm of Schmid, decomposed by SVD a Krylov matrix:

$K_{0}^{N-1}=U \Sigma V^{H}$

is introduced into the equation $K_{0}^{N-1}=K_{1}^{N}$ :

$A U=K_{1}^{N} V \Sigma^{-1}$

with $K_{1}^{N}:=\left\{A N_{0}, A N_{1}, A N_{2}, \ldots, A N_{N-1}\right\}$.

Using left side multiplication by $U^{H \text { : }}$

$U^{H} A U=U^{H} K_{2}^{N+1} V \Sigma^{-1} \equiv C$

$C$ is a matrix similar to $A$. Not to be explicitly known (only its action on certain vectors known), this approach allows to be reduced to the approximation of a known linear operator and small size, $C$. The eigenvalues $\lambda_{i}$ of the similar matrix $C$ are eigenvalues of $A$. Consider the eigenvalue of $C$, and $v$ an associated eigenvector:

$A K_{1}^{N} v=K_{1}^{N} C v=K_{1}^{N} \lambda v=\lambda K_{1}^{N} v$

$A\left(K_{1}^{N} v\right)=\lambda\left(K_{1}^{N} v\right)$

Eigenvectors $v_{i}$ of a similar matrix, for example the companion matrix $C$, are therefore deducted from $\Psi_{i}$ eigenvectors of $A$ by:

$\psi_{i}=K_{1}^{N} v_{i}$

\section{Results}

Four cases have been studied for an incidence angle of $10^{\circ}$, a velocity of $5 \mathrm{~ms}^{-1}$ and four values of the cavitation index of 2 (steady cavity), 1.75 (periodic cavity), 1.5 (transitional cavity) and 1.25 (aperiodic cavity).

\subsection{Space-time diagram}

\subsubsection{Quasi-steady cavity $(\sigma=2)$}

Even if cavitation is never a totally steady phenomenon, a quasisteady configuration is presented on. It can be seen that a sheet 

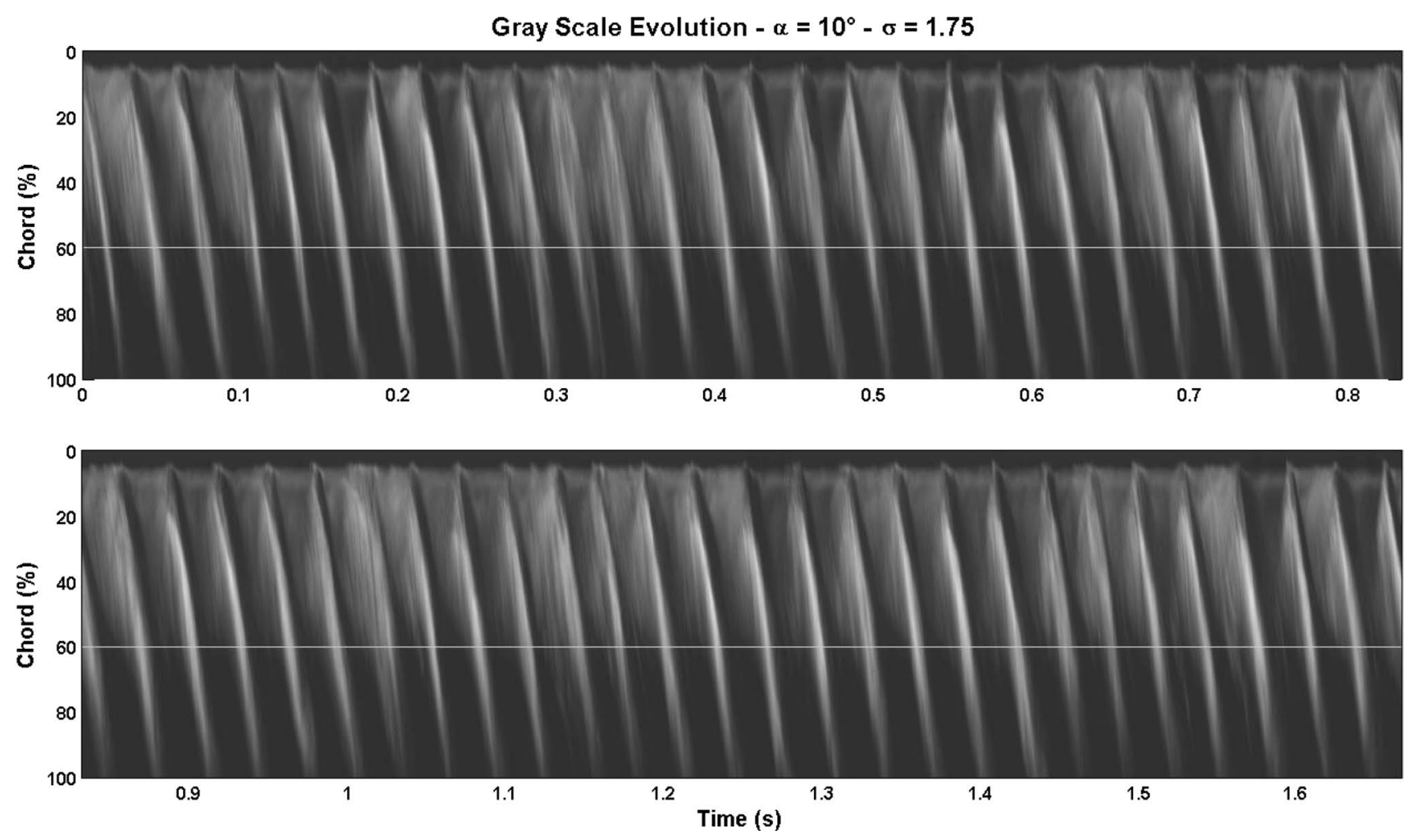

Fig. 7 Space-time diagram $\left(\sigma=1.75, \alpha=10^{\circ}\right)$

cavity of roughly $30 \%^{2}$ remains attached near the leading edge of the foil. Vapor clouds torn off the cavity can be identified periodically in the cavity wake at about $40 \mathrm{~Hz}$. The modification of the cavity length remains small. At certain moment, a more fluctuating behavior can be seen (areas surrounded by white frames). During these time intervals, the shedding is intensified and the associated frequency is evaluated at $50 \mathrm{~Hz}$. A Fourier transform has been applied to extract the main frequencies leading to the frequency diagram of Fig. 10d. On this diagram, a peak can be identified at $46.9 \mathrm{~Hz}( \pm 0.1 \mathrm{~Hz})$.

\subsubsection{Periodic cavity $(\sigma=1.75)$}

In this case, the cavity behaves periodically and a total disappearance of the cavity can be observed in Fig. 7 when the reentrant jet cuts off the cavity at the leading edge. Moreover, a direct estimation of the velocity of the cavity leading edge and trailing edge can be carried out following the procedure previously described. Even if the

\footnotetext{
${ }^{2}$ All the cavity length presented are made no dimensional by the chord length of the profile.
}

periodicity of the phenomenon is well established, the evaluation of the velocities gives rise to large uncertainty. These measurements will be carried out again on the phaseaveraged reconstruction of the cavity behavior. On the frequency diagram, the main shedding is clearly identified at $f_{\mathrm{s}}=32.6 \mathrm{~Hz}$ in Fig. 10c.

\subsubsection{Transitional cavity $(\sigma=1.5)$}

For a lower value of $\sigma$, the periodical behavior of the cavity is slightly perturbed, Fig. 8. A main shedding is clearly identified at $f_{\mathrm{s}}=22.6 \mathrm{~Hz}$ in Fig. $10 \mathrm{~b}$, but the spectral peak has broadened, corresponding to the appearance of a pseudo-periodicity.

\subsubsection{Aperiodic cavity $(\sigma=1.25)$}

In Fig. 9a, lowering of the cavity frequency shedding can be observed. Each period is composed of a growing phase followed by a period during which the foil is completely wet. A complex behavior can be observed in the vapor phase associated with the progressive shedding of the cavity. During this 

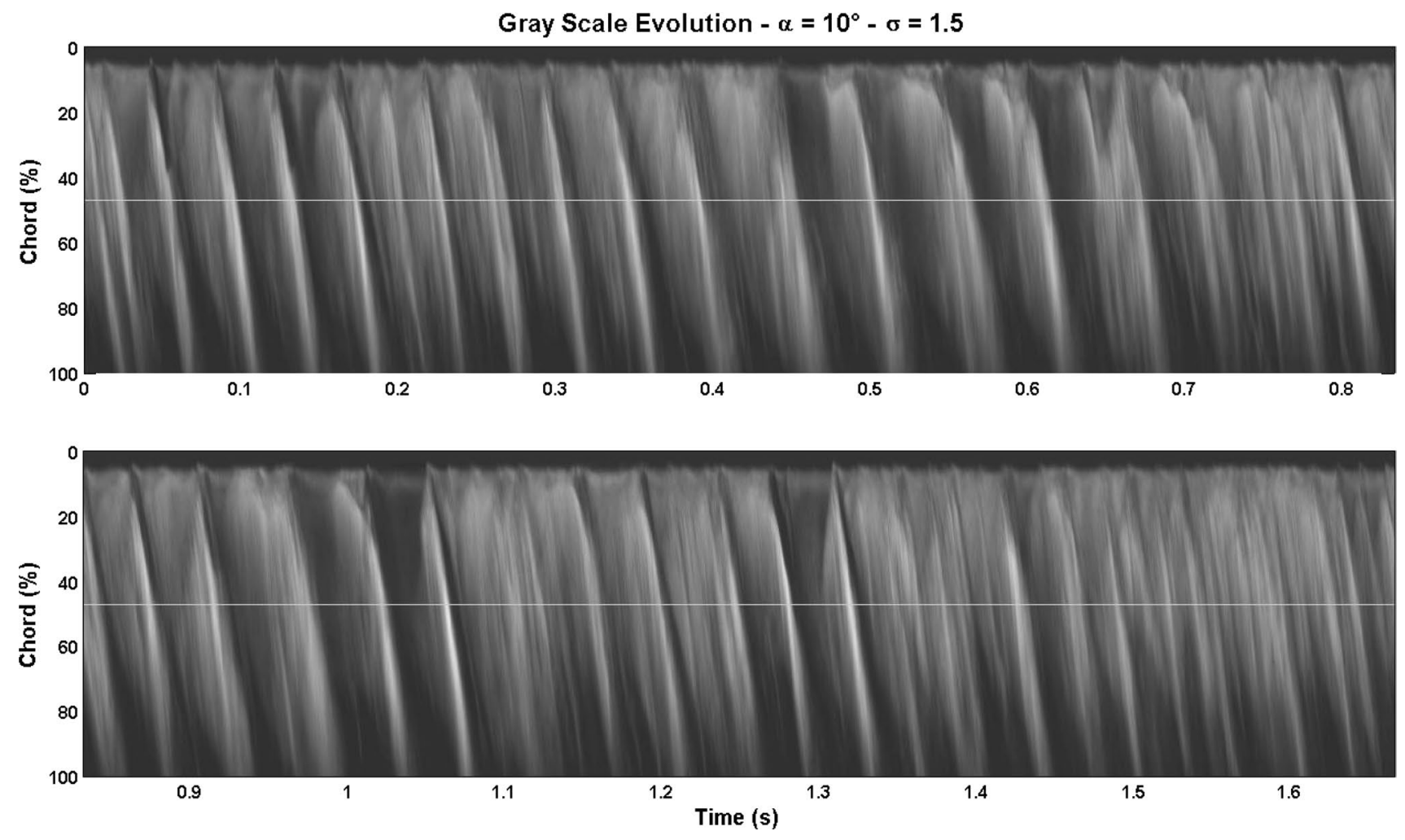

Fig. 8 Space-time diagram $\left(\sigma=1.50, \alpha=10^{\circ}\right)$
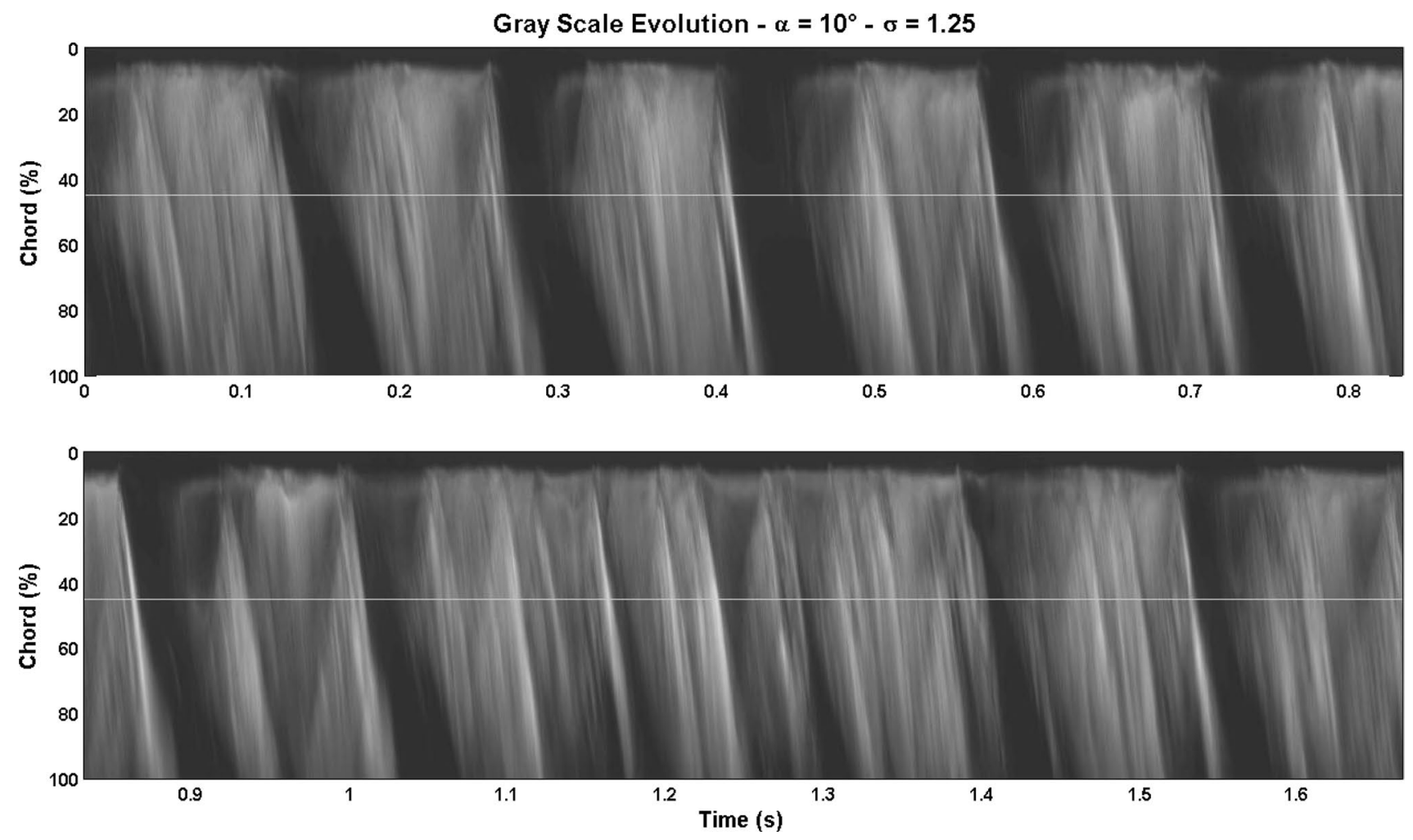

Fig. 9 Space-time diagram $\left(\sigma=1.25, \alpha=10^{\circ}\right)$ 


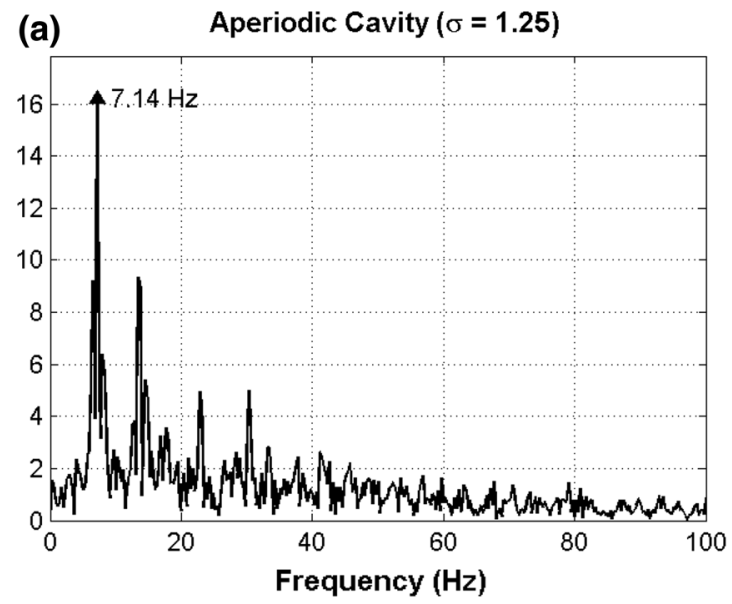

(c)

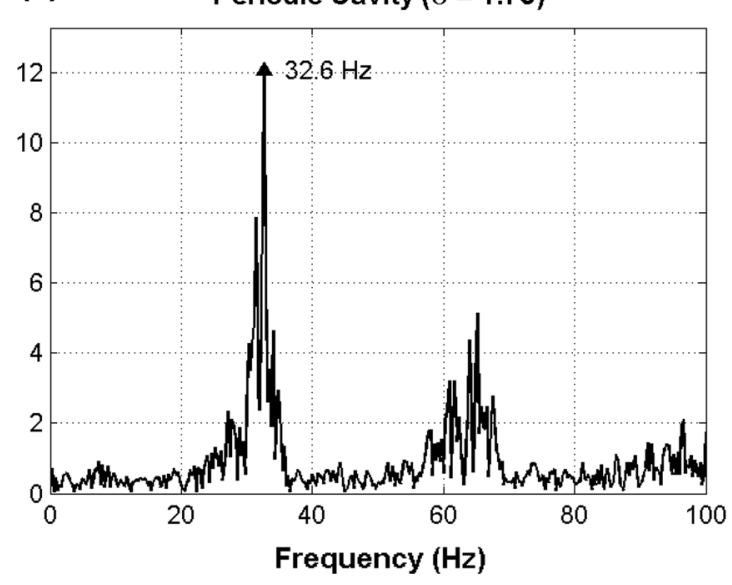

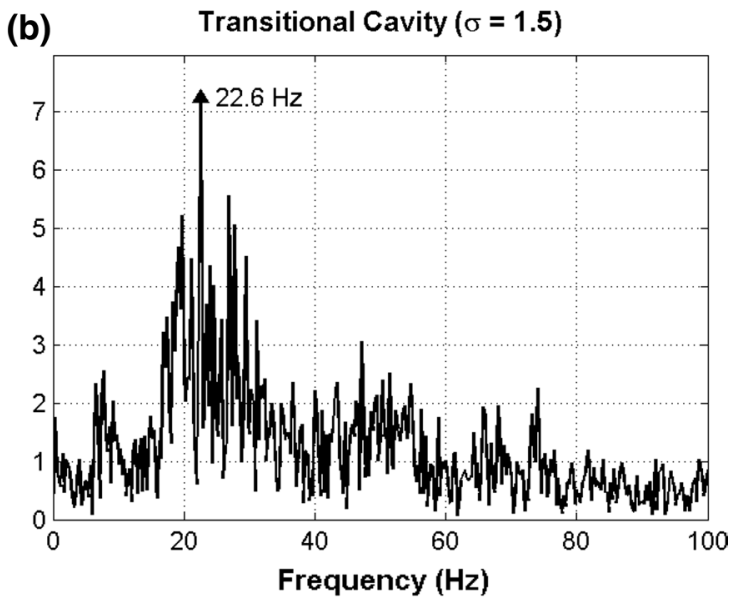

(d) Quasi Steady Cavity $(\sigma=2)$

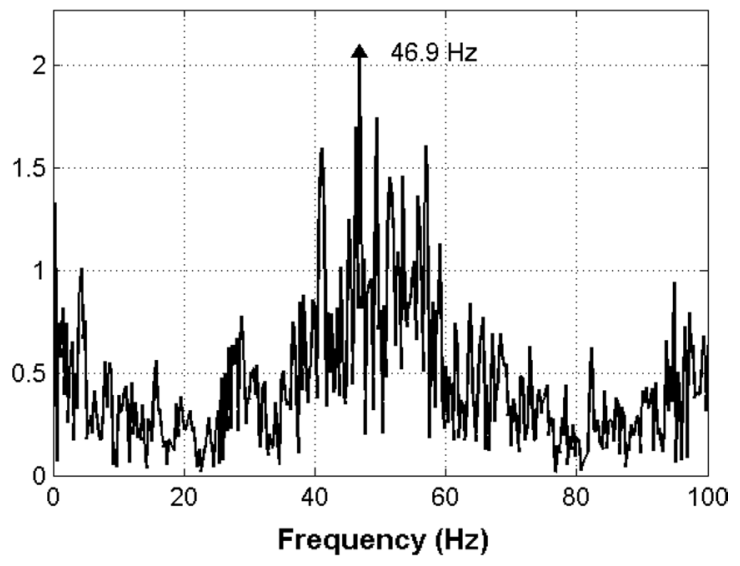

Fig. 10 Frequency diagram $\left(\alpha=10^{\circ}\right)$. a $\sigma=1.25, \mathbf{b} \sigma=1.5$, $\mathbf{c} \sigma=1.75, \mathbf{d} \sigma=2$

phase, we took instantaneous pictures of the cavity, presented in Fig. 11, which exhibit a 3D squared pattern which cannot be assessed by the time-averaged procedure. A frequency analysis has been conducted on this data set, and the main shedding frequency is identified at $f_{\mathrm{s}}=7.14 \mathrm{~Hz}$ (Fig. 10a).

$\mathrm{St}_{c}=\frac{f_{\mathrm{s}} \cdot c}{U_{\infty}} \quad \mathrm{St}_{l c}=\frac{f_{\mathrm{s}} \cdot l c}{U_{\infty}}$

The results are summarized in Table 1 .

To sum up, for $\sigma / 2 \alpha>4$ (corresponding to $\sigma=2$ ) the cavity length and the Strouhal number corroborate the results of Arndt et al. (2000) and Kawakami et al. (1997) where the cavity is attached near the leading edge with few vapor clouds periodically ripped off. The behavior of the cavity is quasi-periodic. For $\sigma=1.75(\sigma / 2 \alpha=5)$, the vapor cavity presents a periodic behavior and the cavity totally disappears when the reentrant jet cuts the cavity near the leading edge. The dynamics of the sheet cavity are governed by the liquid jet. When $\sigma / 2 \alpha=4.3(\sigma=1.75)$, a pseudo-periodical (or transitional) behavior can be observed where the shedding process is irregular, corresponding to the transition instability area. For the last studied value of $\sigma / 2 \alpha<4(\sigma=1.25)$, the shedding frequency decreases notably and each cycle is constituted by growth phase of the cavity followed by wetting of the foil without any vapor phase. The behavior of the cavity is aperiodic. The Strouhal number decreases, and the preponderant physical mechanism is the shock wave propagation at the interface of the pocket.

\subsection{Application of POD on sheet cavity pictures}

In the previous section, it was assumed that the behavior of the cavity could be represented by a two-dimensional 

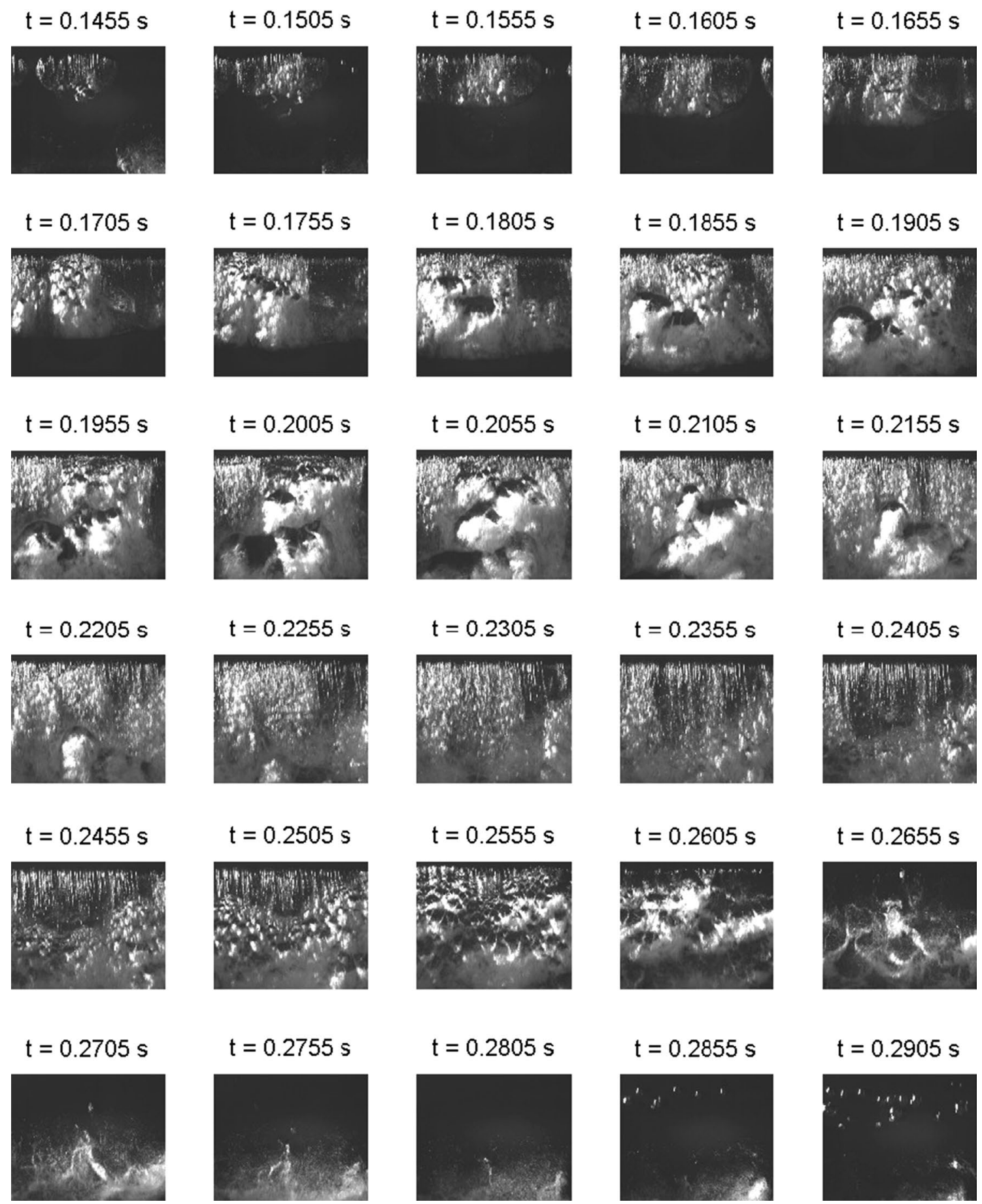

Fig. 11 Snapshots corresponding to a cycle of shedding cavity $\left(\sigma=1.25, \alpha=10^{\circ}\right)$

Table 1 Shedding parameters

\begin{tabular}{llllllll}
\hline Case & $\alpha\left(^{\circ}\right)$ & $\sigma$ & $\sigma / 2 \alpha$ & $L_{\mathrm{c}} / C$ & $f_{\mathrm{s}}(\mathrm{Hz})$ & $\mathrm{St}_{c}$ & $\mathrm{St}_{l c}$ \\
\hline Steady cavity & 10 & 2 & 5.73 & 0.16 & 46.9 & 0.94 & 0.15 \\
Periodic cavity & 10 & 1.75 & 5.01 & 0.51 & 32.6 & 0.65 & 0.33 \\
Transitional cavity & 10 & 1.50 & 4.30 & 0.56 & 22.6 & 0.45 & 0.25 \\
Aperiodic cavity & 10 & 1.25 & 3.58 & 0.54 & 7.14 & 0.14 & 0.08 \\
\hline
\end{tabular}



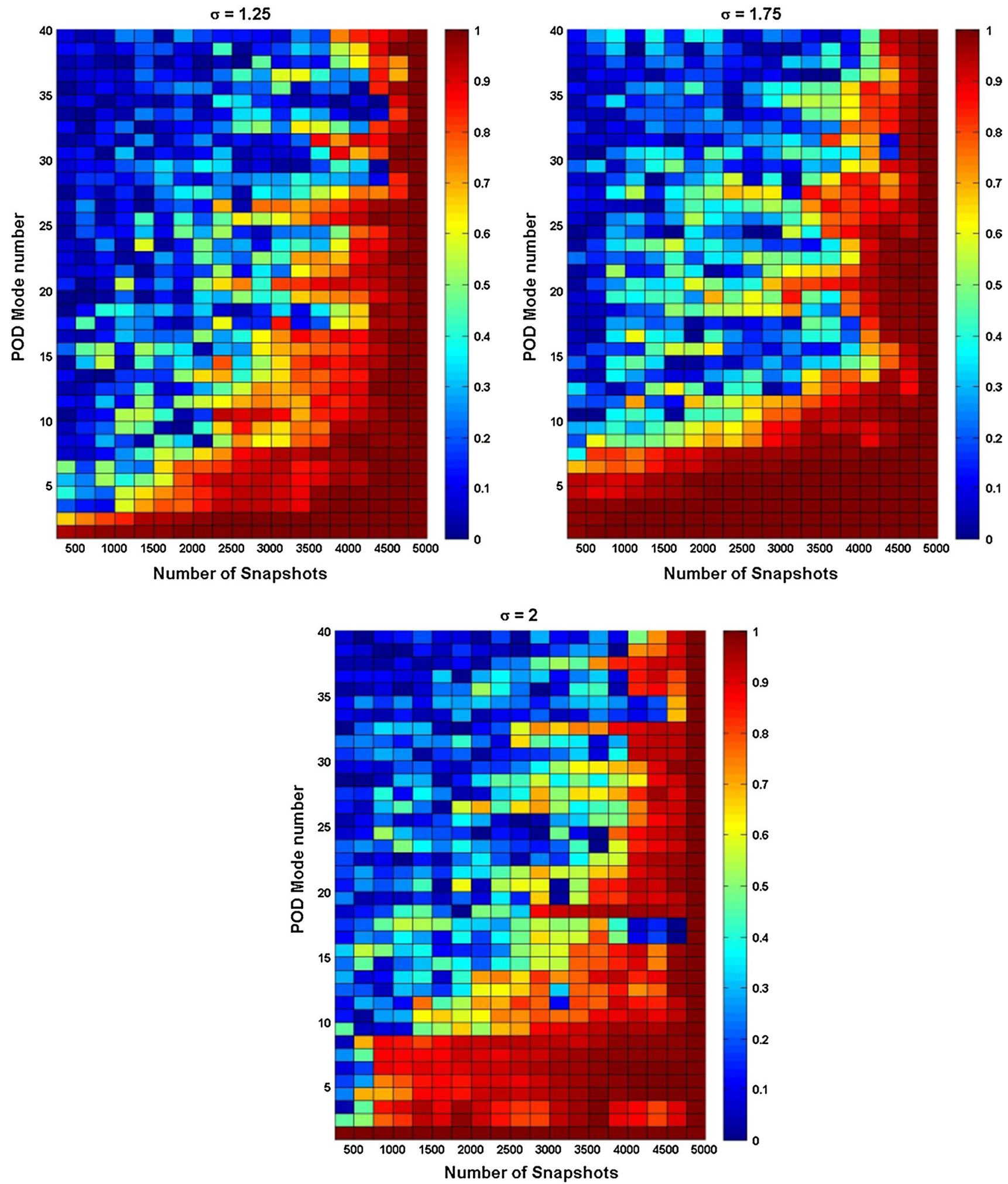

Fig. 12 POD convergence diagram for the tree data set 
Fig. 13 POD Energy distribution, for the first twenty modes, for the tree data set

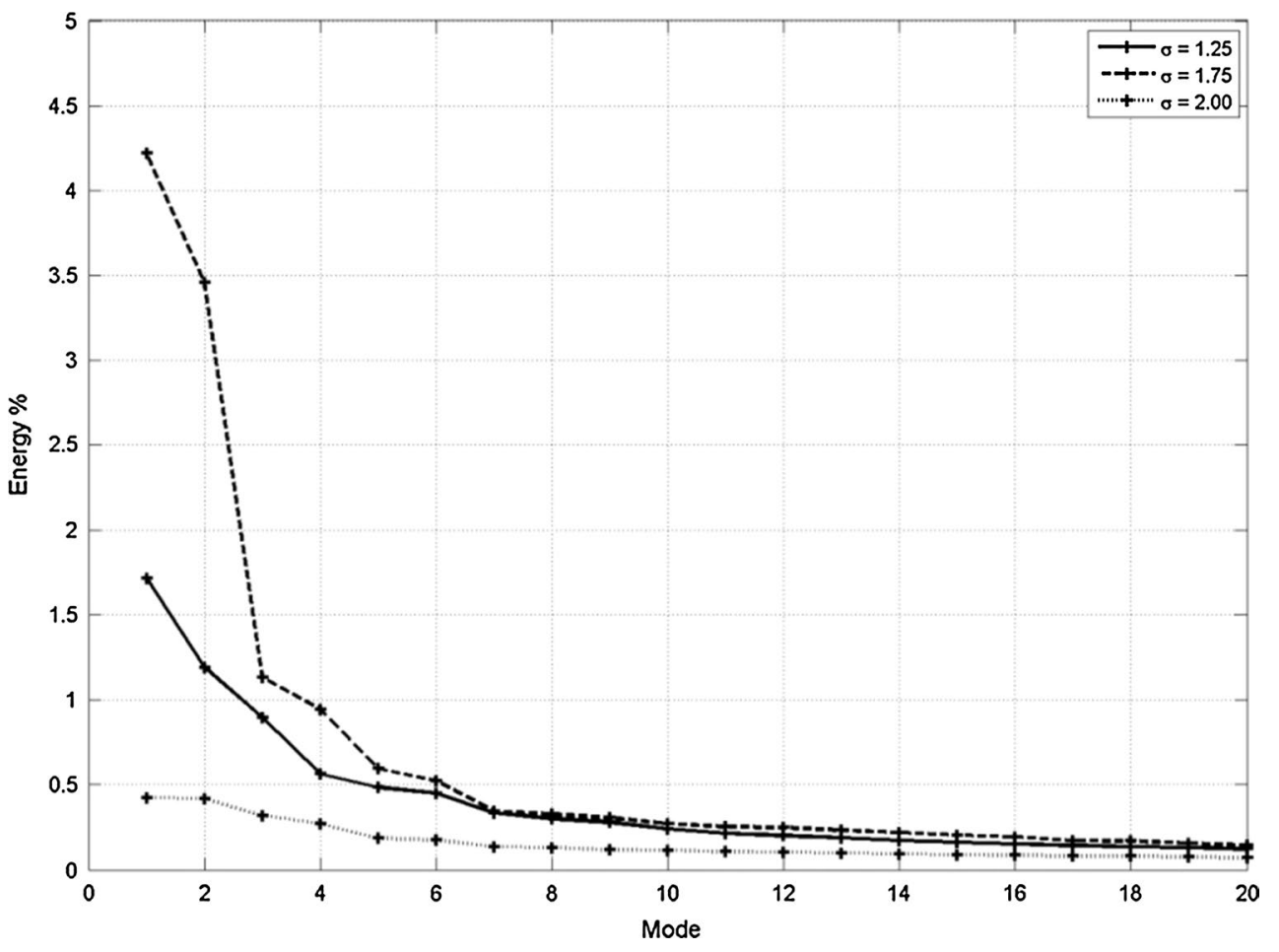

mean value. To get a better understanding of the cavity dynamics and to identify the two-dimensional component of the dynamics from the three-dimensional one, it has been decided to apply the POD described above on the fluctuating part of the signal for the three studied cases $(\sigma=2,1.75,1.25)$. In POD analysis of periodic flows, the first mode (mode 0 ) is associated with the mean flow and suppressed in the present analysis. It represents 87, 75 and $82 \%$ of the total energy of the flow, respectively; the largest value corresponds to most stable case obtained for $\sigma=2$ (quasi-steady). The number of POD modes to be used for the following analysis depends on the rate of convergence of the POD procedure. To test the convergence rate of the POD process, a method is proposed by Hekmati et al. (2011). A convergence matrix $C_{n_{\text {samples }}^{(n)}}$ of the three data sets is presented in Fig. 12. From this figure, and a threshold value of 0.7 for the correlation coefficient, it can be seen that 10 modes are converged for data sets of 3500 snapshots. Thus, we can trust the result obtained for those 10 first modes for the complete data set of 5000 images.

\subsubsection{Direct analysis of the POD decomposition}

3.2.1.1 Eigen values The percentage of energy contained in the 10 first modes for the three data sets is presented in
Fig. 13. In the quasi-periodic case, the energy is regularly spread on the ten modes with a very small increase corresponding to modes $1-4$. The two remaining cases differ in modes 1 and 2 which are reinforced in the periodic case where an important percentage of the total energy is spread on those two first modes. In the unsteady cases $(\sigma=1.75$, 1.25) modes 1 and 2 are predominant. It can be seen in Fig. 14 that the greater the unsteadiness the larger, the predominance of lower modes and that for modes 7 and larger the percentage of energy is equivalent. The topology of the spatial modes will indicate the two or three dimensionality of the cavity behavior.

3.2.1.2 Spatial modes The spatial modes associated with the three data sets are presented in Fig. 14a-c. In these figures, each inset corresponds to the topology of the mode, in a space of a one chord by one chord. The top of each inset corresponds to the leading edge and down to the trailing edge. In the quasi-steady case, modes 1-4 have a correct two-dimensional aspect while modes 5 and 6 remain rather two-dimensional and all these modes are associated with a low value of the energy. In this case, the POD analysis leads to a bi-dimensional mean governed behavior. In the periodic case, modes $1-4$ are strongly periodic and two-dimensional, with a spatial frequency doubling on modes 3 and 4. Associ- 
(a)

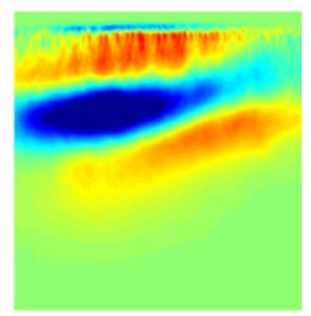

Mode 1

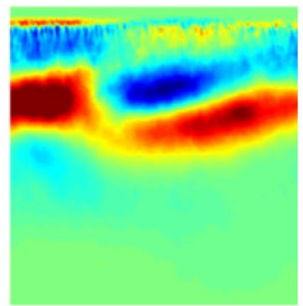

Mode 3

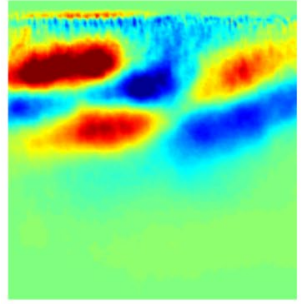

Mode 5

(c)

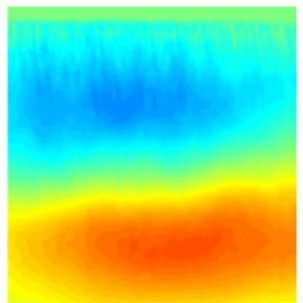

Mode 1

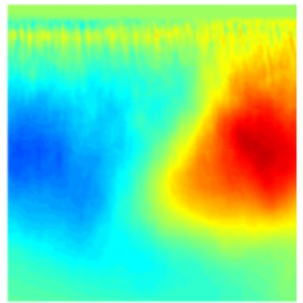

Mode 3

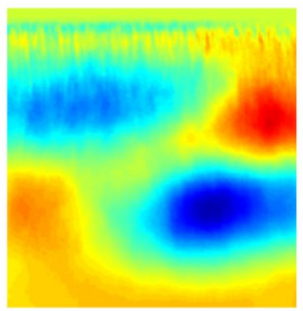

Mode 5

(b)

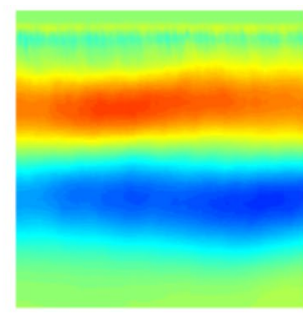

Mode 1

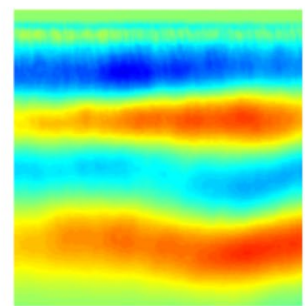

Mode 3

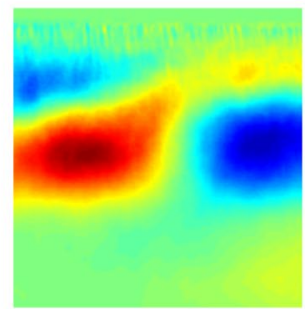

Mode 5

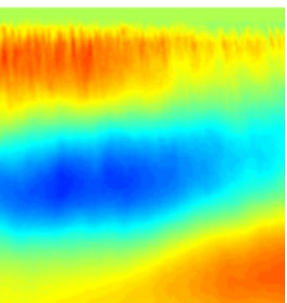

Mode 2

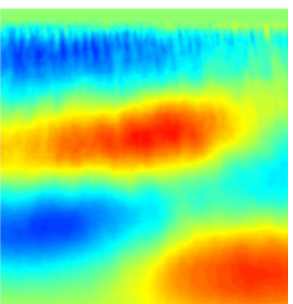

Mode 4

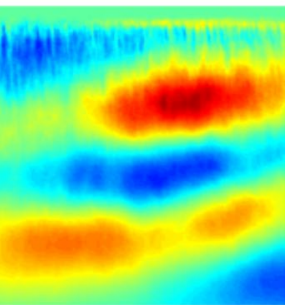

Mode 6

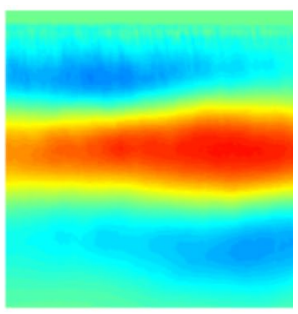

Mode 2

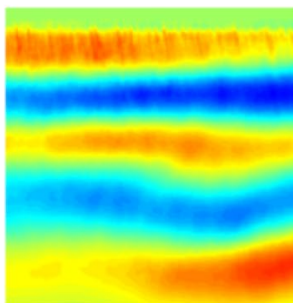

Mode 4

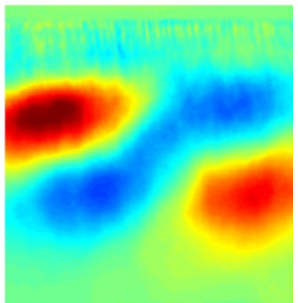

Mode 6 
Fig. 15 First six POD temporal coefficients $\mathbf{a} \sigma=2, \mathbf{b} \sigma=1.75$ and $\mathbf{c} \sigma=1.25$
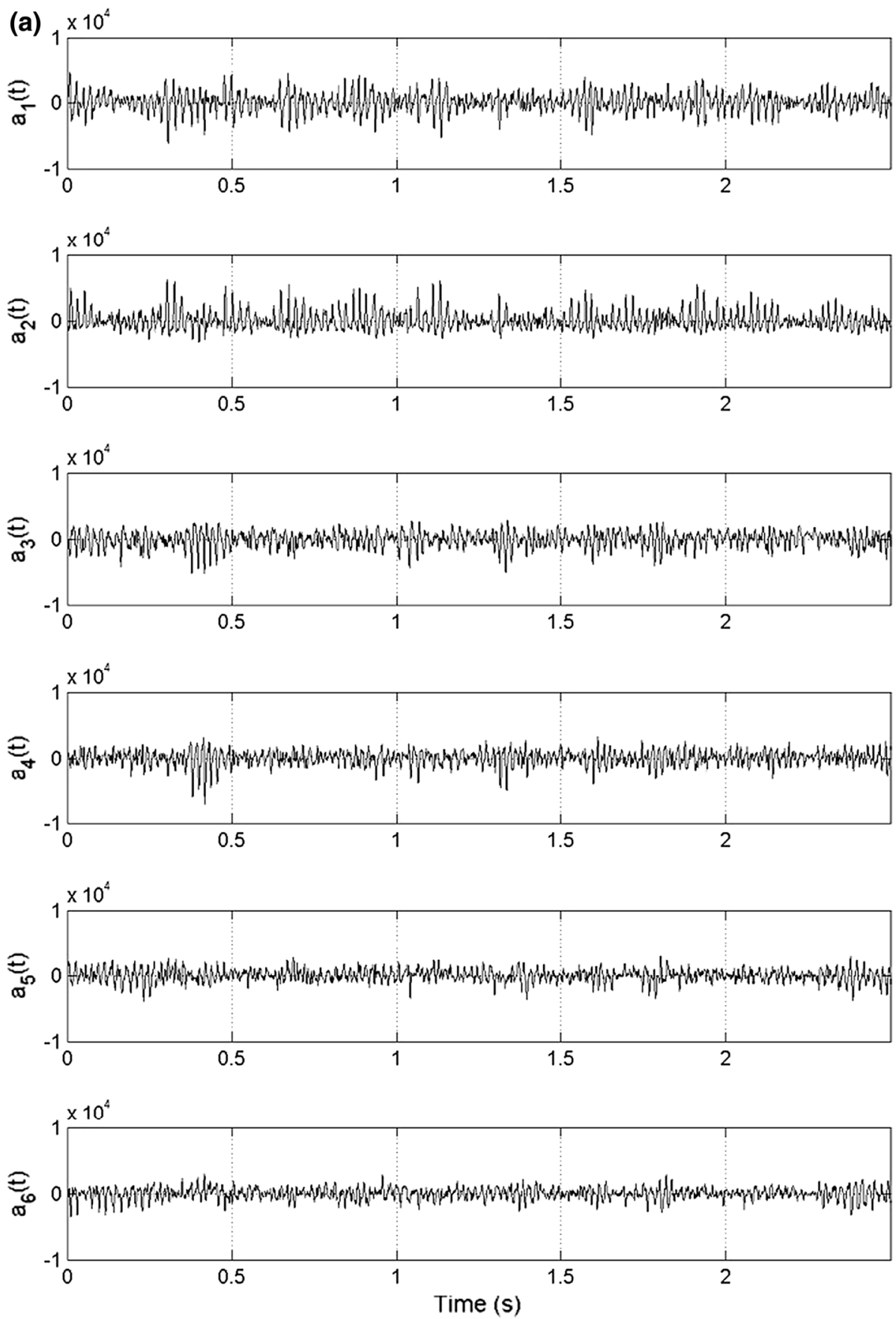

ated with a large value of the energy associated with modes 1 and 2, this case is clearly dominated by the main cavity shedding. In the third case, the two-dimensional behavior can only be identified in mode 1 and 2 . All the other modes are three-dimensional, and a more complex behavior is expected. This will be investigated further. The spatial fre- quency is obviously increased, and the three dimensional modes (modes 3-6) are energetic enough to modify the visual aspect of the cavity which can no longer be assumed as two-dimensional. For $\sigma=2$, the cavity presents a quasisteady behavior. The cavity length is small, and the contributions of each mode from 1 to 6 are smaller compared to 
Fig. 15 continued
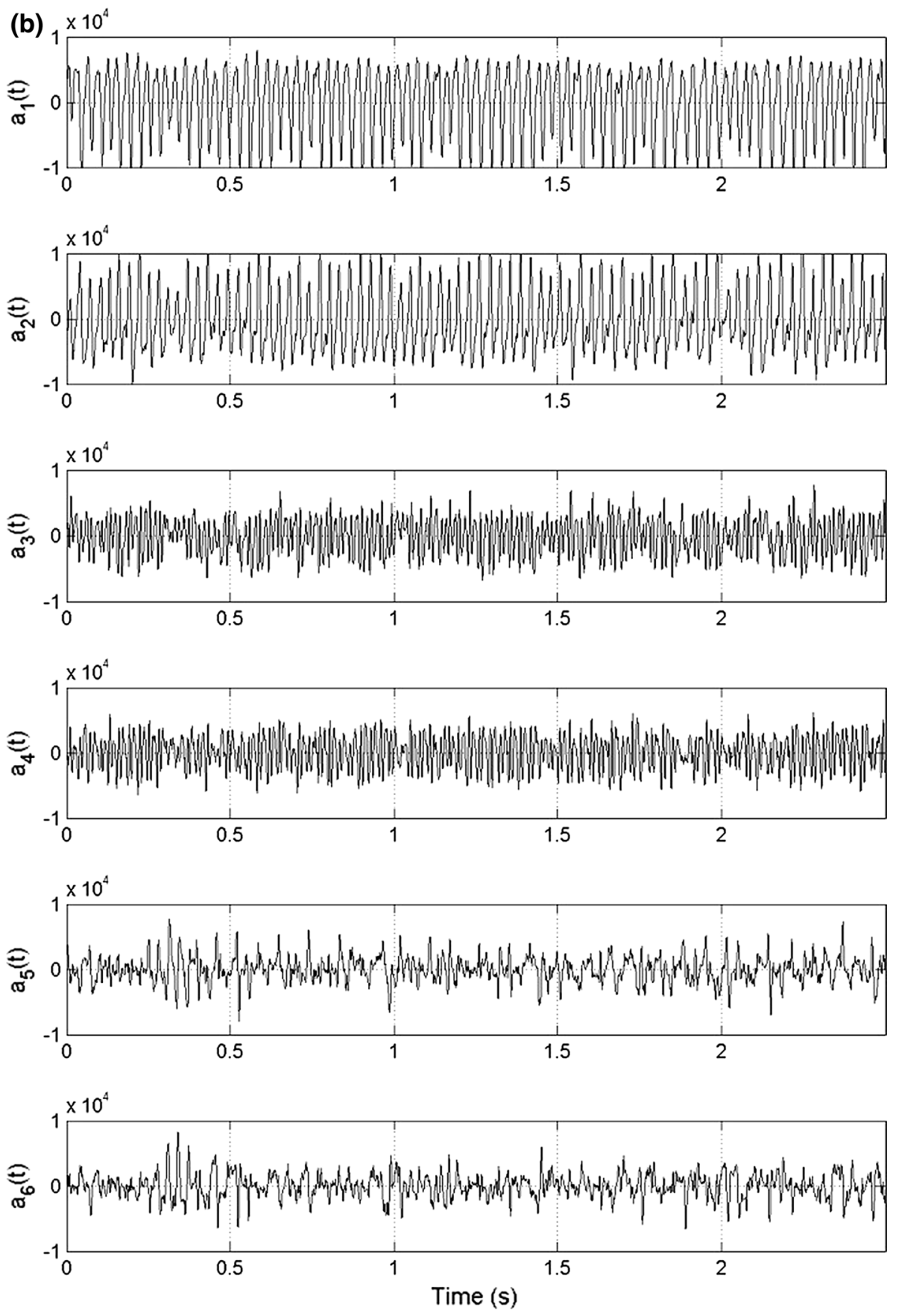

the case corresponding to smaller sigma value. In this case, the periodicity is not clearly detected due to the small contribution of energy (Fig. 13).

3.2.1.3 Temporal coefficients The temporal coefficients $a_{i}(t)$ associated with modes 1-6 are presented in Fig. 15a- c. In the first case $(\sigma=2)$, the amplitude of the modes is equivalent. Modes 1 and 2 exhibit gusts during which the amplitude of the temporal coefficients increases. These gusts attest to the existence of a low frequency around $4 \mathrm{~Hz}$ visible on the space frequency diagram shown in Fig. 10d. In the second case $(\sigma=1.75)$, the four first modes have 
Fig. 15 continued
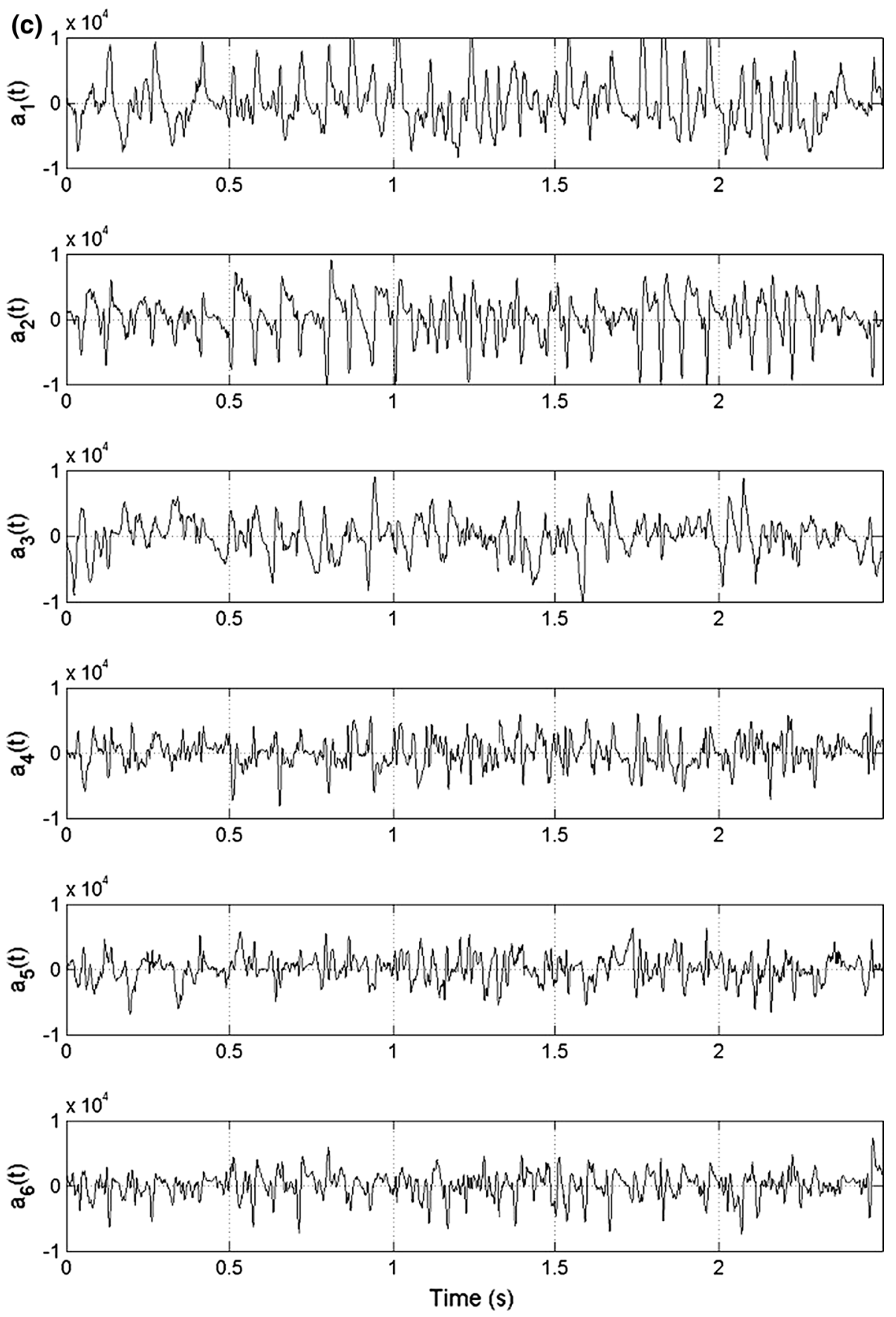

a well-defined periodicity with a frequency doubling concerning modes 3 and 4 . The frequency of modes 1 and 2 corresponds to the main shedding frequency of the cavity defined in Sect. 3.1.2. The energy of modes 3 and $4\left(2 * f_{\mathrm{s}}\right)$ represents in that case $27 \%$ of the energy of modes 1 and 2 $\left(f_{\mathrm{s}}\right)$. Based on periodicity of modes 1 and 2 , a phase can be defined by: $\tan \left(\phi_{12}\right)=\frac{a_{2} / \sigma_{a_{2}}}{a_{1} / \sigma_{a_{1}}}$

In Fig. 16, the temporal coefficients are reported versus $\varphi_{12}$ values. It can be seen that modes 1 and 2 on one hand and modes 3 and 4 on the other hand are in phase quadrature. The frequency doubling can also be seen on this 
Fig. 16 First six POD temporal coefficients versus $\varphi_{12}$ value, for $\sigma=1.75$
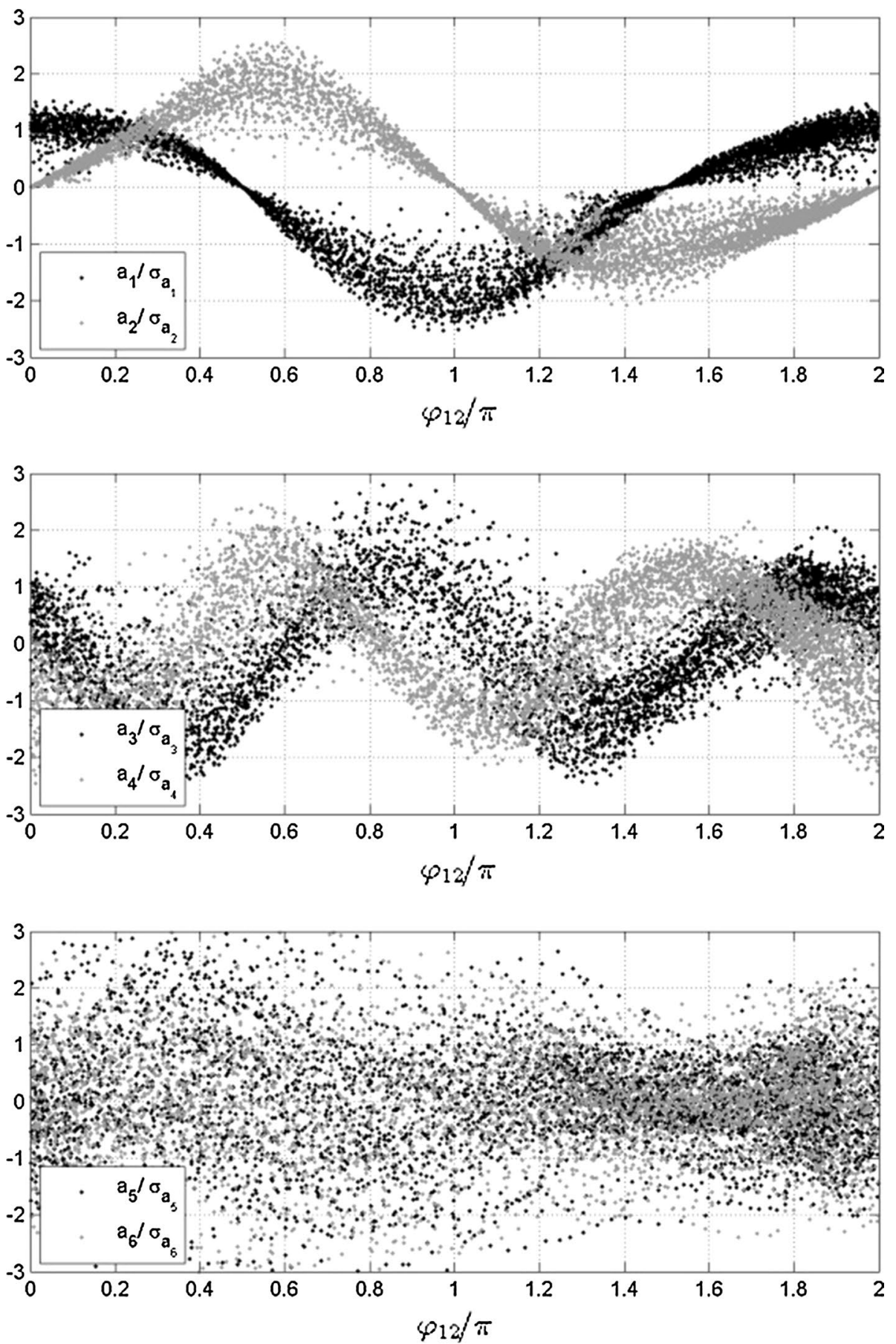

representation. It is difficult to give a physical interpretation for the higher modes. This phase will be used for a further phasing of the phenomenon. In the third case $(\sigma=1.25)$, the temporal coefficients have a more complex behavior where the shedding frequency can be identified. The complexity of the signal and its lack of periodicity suggest a chaotic behavior of the phenomenon in this case. This point requires further analysis. 


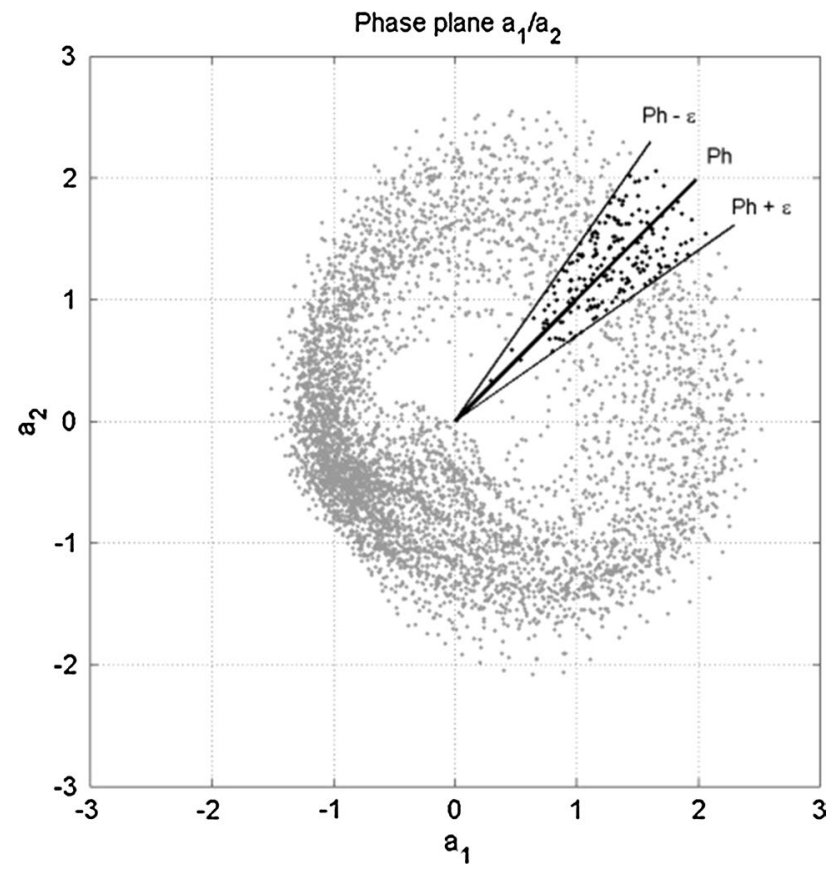

Fig. 17 Phase plane $\left(a_{1} / \sigma_{1}, a_{2} / \sigma_{2}\right)$ for $\sigma=1.75$

\subsubsection{Dynamic behavior of the cavity analysis}

In this paragraph, only the periodic case $(\sigma=1.75)$ will be studied. The POD decomposition will be used to phase the cavity shedding to reconstruct a phase average representation of the phenomenon.

\subsubsection{Phase reconstruction A representation of a tem-} poral coefficient $a_{i}$ as a function of a second temporal coefficient $a_{j}$ leads to Lissajous figures (Oberleithner et al. 2011), Fig. 17. The choice of snapshots to be used for each phase average will be taken from the phase value defined by the POD temporal coefficient, $\varphi_{12}$ shown in Fig. 16. For the following reconstruction, the phase values have been chosen every $5^{\circ}$ with a width value of $4^{\circ}$. For example in Fig. 18, 20 snapshots corresponding to a phase of $45^{\circ}$ are represented. It can be seen that the POD phase allows a pertinent choice of the pictures corresponding to the same position in the shedding process.

\subsection{Cross discussion on POD and DMD analysis}

If we observe the distribution of values for the four selected configurations, in the pseudo-periodic case $(\sigma / 2 \alpha=5.73)$, the POD eigenvalues, corresponding to the energy of the flow, are evenly distributed on all the modes, with a very slight increase in modes $1-4$. Other cases differ in modes 1 and 2, which are reinforced with the periodicity of the flow, where a significant percentage of the total energy is left on the first modes. We show that for $\sigma / 2 \alpha=5$, the first two modes are the most energetic, corresponding to a release of periodic vapor structures. In the POD analysis (which is also true for DMD), mode 0 is associated with the mean flow (with $87,75,79,82 \%$ of energy, respectively). For the higher-order modes, mode 1 comprises a two-lobe counter-rotating structure characteristic of spatial periodic detachment. Mode 2 represents the same structures with a delay in downstream direction of about a quarter wavelength. This is in agreement with numerical and experimental studies for various Reynolds numbers in the case of bluff body wake configurations (Noack et al. 2003; Van Oudheusden et al. 2005; Perrin et al. 2007). The largest value is for $\sigma / 2 \alpha=5.73$ (stationary case), and the lowest value is for $\sigma=1.75$, with a predominance of the first two modes. Given the energy signature of the modes, it may be noted that a small number of modes may be enough to describe the dynamics of the sheet in our experiment.

In Fig. 19, DMD spectra are presented for $\sigma / 2 \alpha=5.73$, 5, 4.3 and 3.58. As regards the DMD spectra, it is customary to draw the real part of the L2 standard modes $\Psi_{i}$ as a function of the frequency $\omega_{i}^{k}$, defined by:

$\omega^{k}=\frac{\ln \lambda_{k}}{2 \pi \Delta t}=\omega_{r}^{k}+i \omega_{i}^{k}$

Figures 20, 21, 22 and 23 show the first four POD modes $\left(\Phi^{i}\right)$ and the real parts of the DMD modes $\left(\Psi_{r}^{i}\right)$, respectively, for $\sigma / 2 \alpha=5.73,5,4.3$ and 3.58. Regarding POD modes, mode 1 is composed of counter-rotating structures that characterize the spatial periodic detachment of the sheet along the chord. Mode 2, meanwhile, is a classical spatial shift of the first mode (Ma and Karniadakis 2002). This is significant for configuration $\sigma / 2 \alpha=5$. For a larger value of 5.73 , the same behavior is observed with a deflection from the axis of symmetry. Mode 3 characterizes a phenomenon of contraction or stretching of the structure, and mode 4 should introduce a phenomenon of asymmetrization characteristic of a three-dimensional instability due to fluctuations in the closure zone of the sheet. For the higher-order modes, the lower energy characterizes the complexity of flow and the energy trace of the small-scale structures that are convected. 


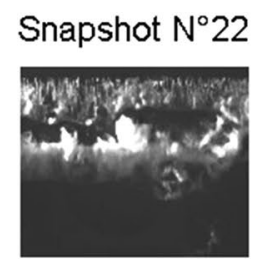

Snapshot $N^{\circ} 440$

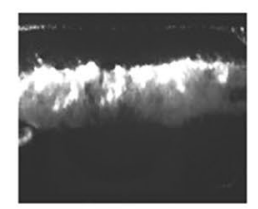

Snapshot N 1042

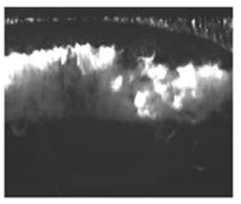

Snapshot $N^{\circ} 1720$

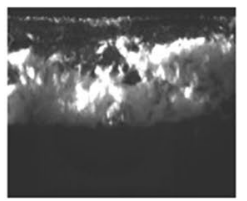

Snapshot $N^{\circ} 2276$

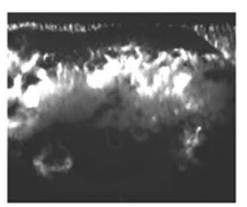

Snapshot $N^{\circ} 2765$

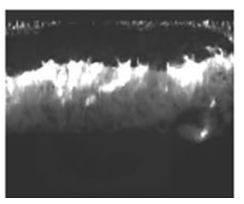

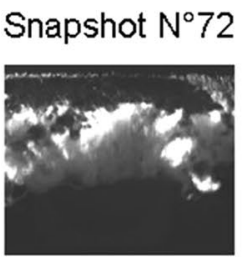

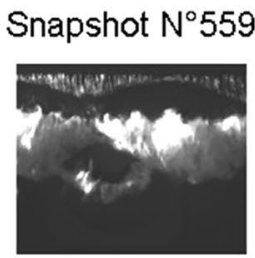

Snapshot N ${ }^{\circ} 1169$

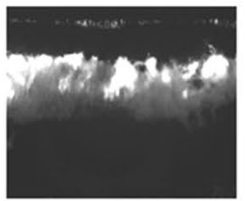

Snapshot $N^{\circ} 1850$

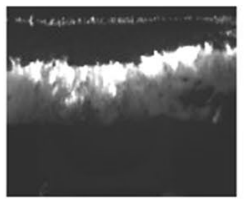

Snapshot N ${ }^{\circ} 2387$

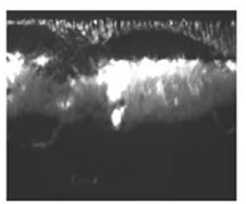

Snapshot N ${ }^{\circ} 2892$

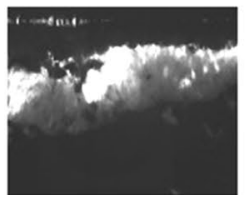

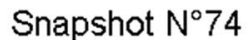
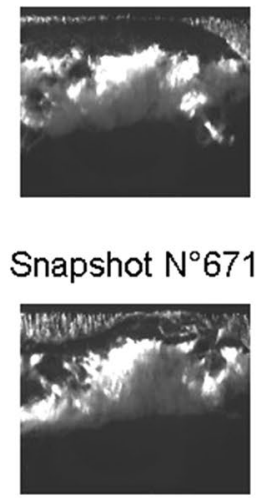

Snapshot N¹294

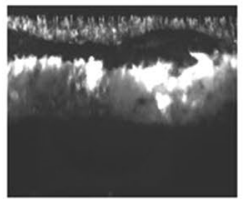

Snapshot N ${ }^{\circ} 1972$

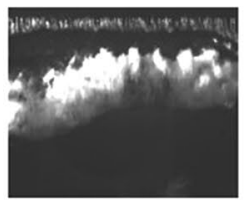

Snapshot N ${ }^{\circ} 2452$

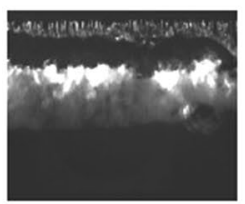

Snapshot N²2951

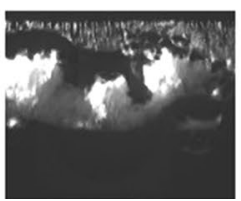

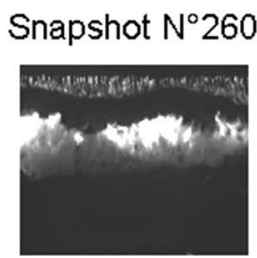

Snapshot N ${ }^{\circ} 796$

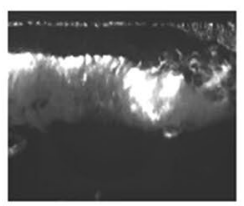

Snapshot $N^{\circ} 1474$

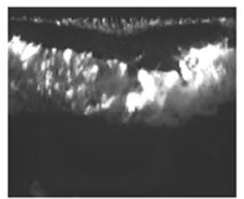

Snapshot N ${ }^{\circ} 2033$

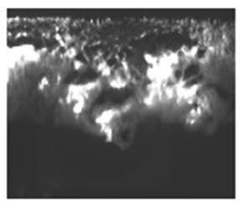

Snapshot N²581

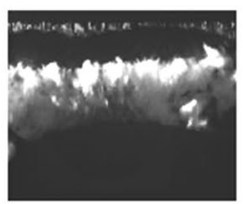

Snapshot N³067

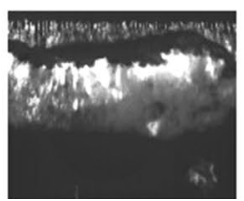

Snapshot $N^{\circ} 377$

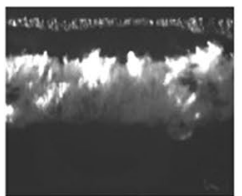

Snapshot $N^{\circ} 913$

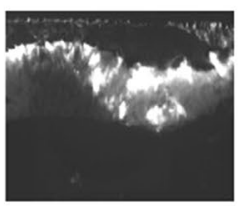

Snapshot $N^{\circ} 1602$

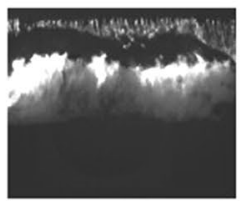

Snapshot $N^{\circ} 2150$

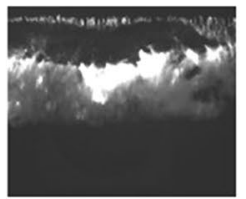

Snapshot N ${ }^{\circ} 2642$

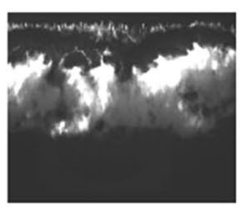

Snapshot N³201

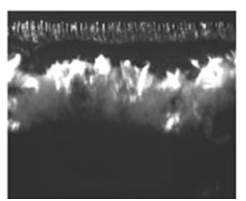

Fig. 18 Chosen snapshots issued from POD analysis

It is thus noted that for all chosen values of $\sigma / 2 \alpha$, and for the first two modes, alternating structures are convected along the quasi-two-dimensional type of profile traces whose energy varies spatially along the chord. However, for the higher-order modes and for $\sigma / 2 \alpha<4$ contraction structures and 3D effects are more pronounced, generating an "asymmetrization" of small-scale structures. This phenomenon is particularly observable for $\sigma / 2 \alpha=4.3$ 

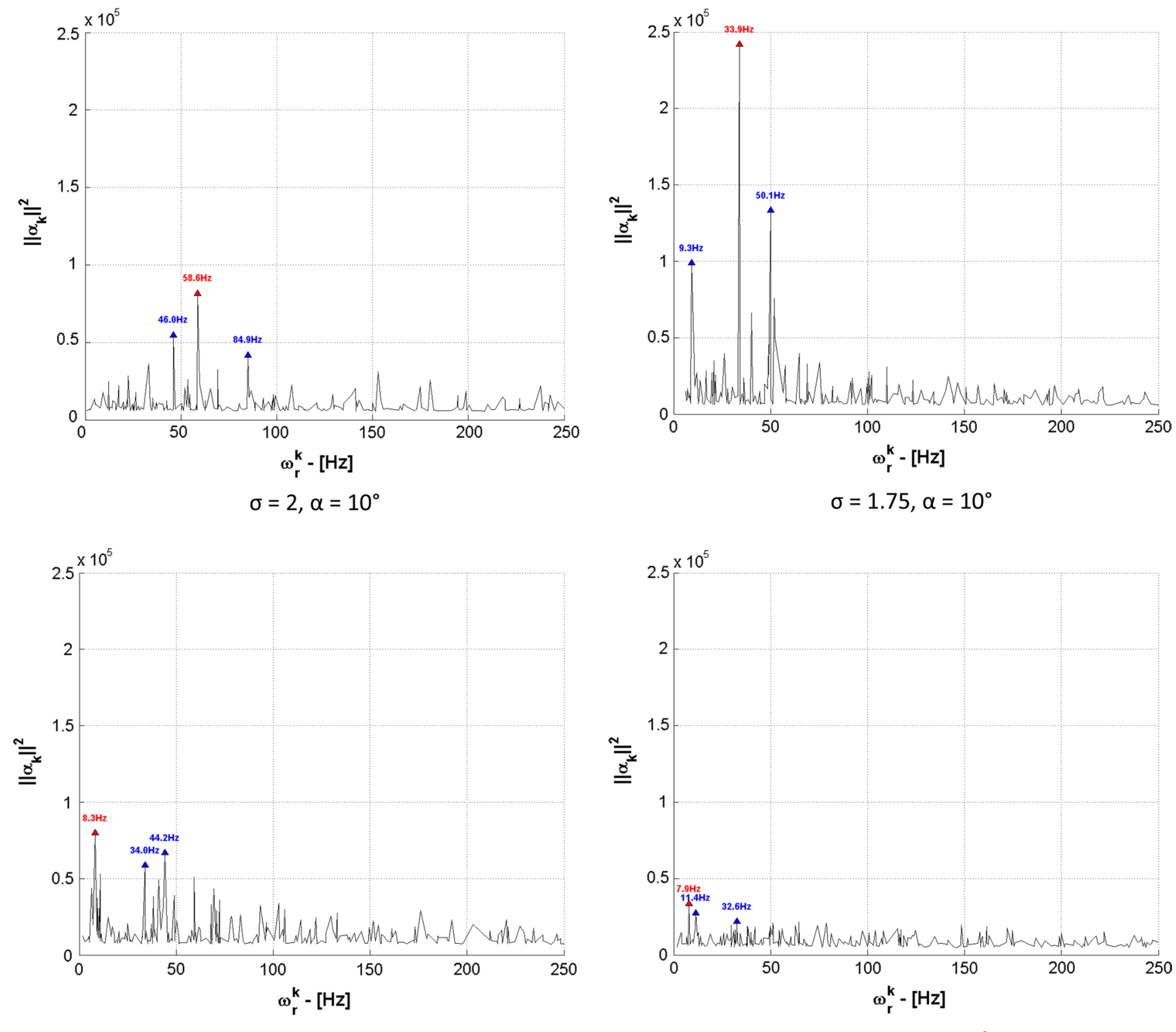

Fig. 19 DMD spectra

corresponding to the transition of the two predominant mechanisms mentioned above (blunt or sharp wave returning) associated with a sudden change of frequency.

DMD applied to high-speed visualizations of the cavitation phenomena allows us to obtain a relationship between the time-frequency oscillations signature pocket and the spatially coherent structures that generate them. This helps to highlight the nonlinear dynamics of the release of pocket transition $\sigma / 2 \alpha=4$ (highlighted by Kjeldsen and Arndt 2001, by a wavelet analysis).

In our particular case, the power spectra obtained by DMD and the associated dynamics spatial modes are shown. It is seen that for values of $\sigma / 2 \alpha>4$, the structures associated with the most energetic frequency peaks are representative of large counter-rotating structures from the periodic release of essentially two-dimensional character 
Fig. 20 Modes $(\sigma=$ $\left.2, \alpha=10^{\circ}\right)$

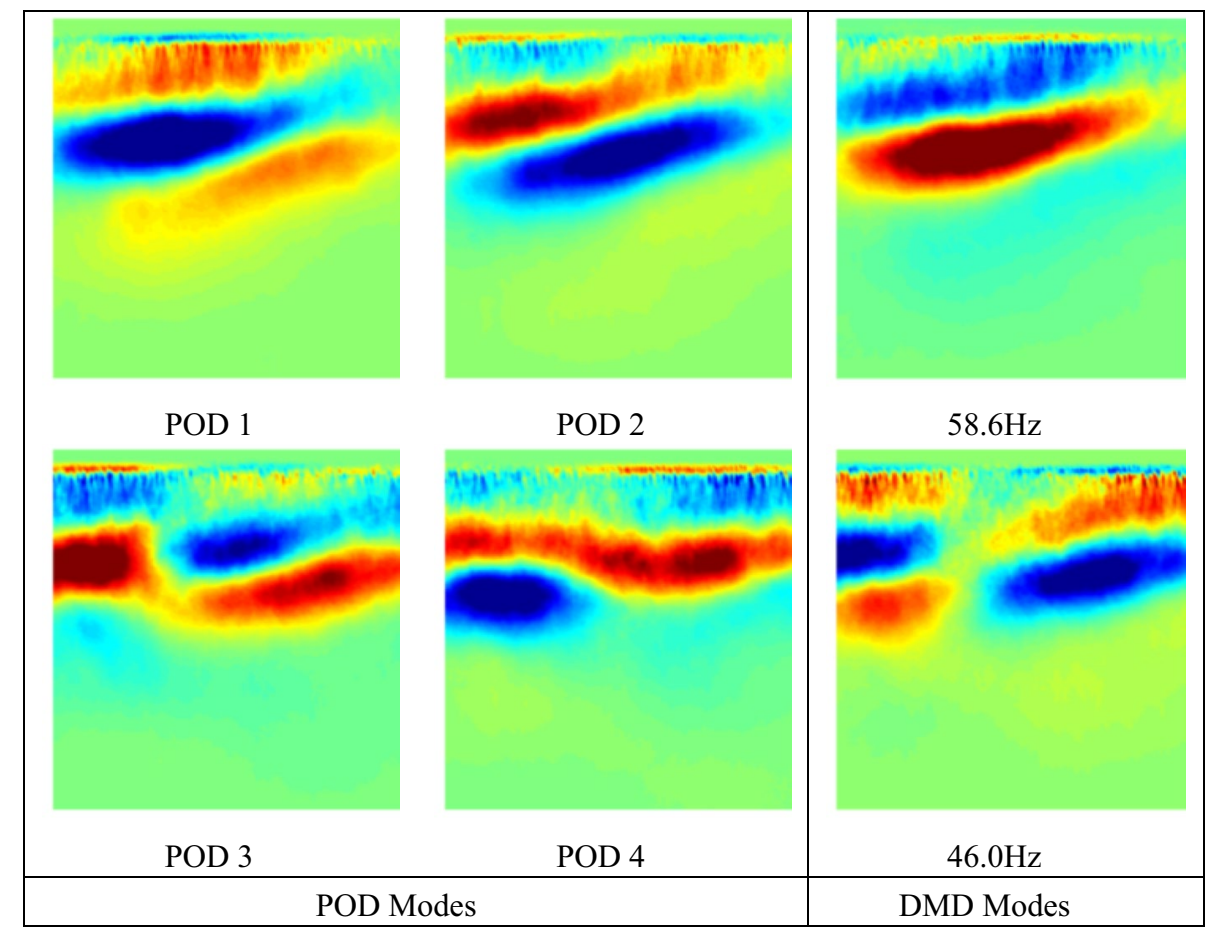

Fig. 21 Modes $(\sigma=1.75$, $\alpha=10^{\circ}$ )

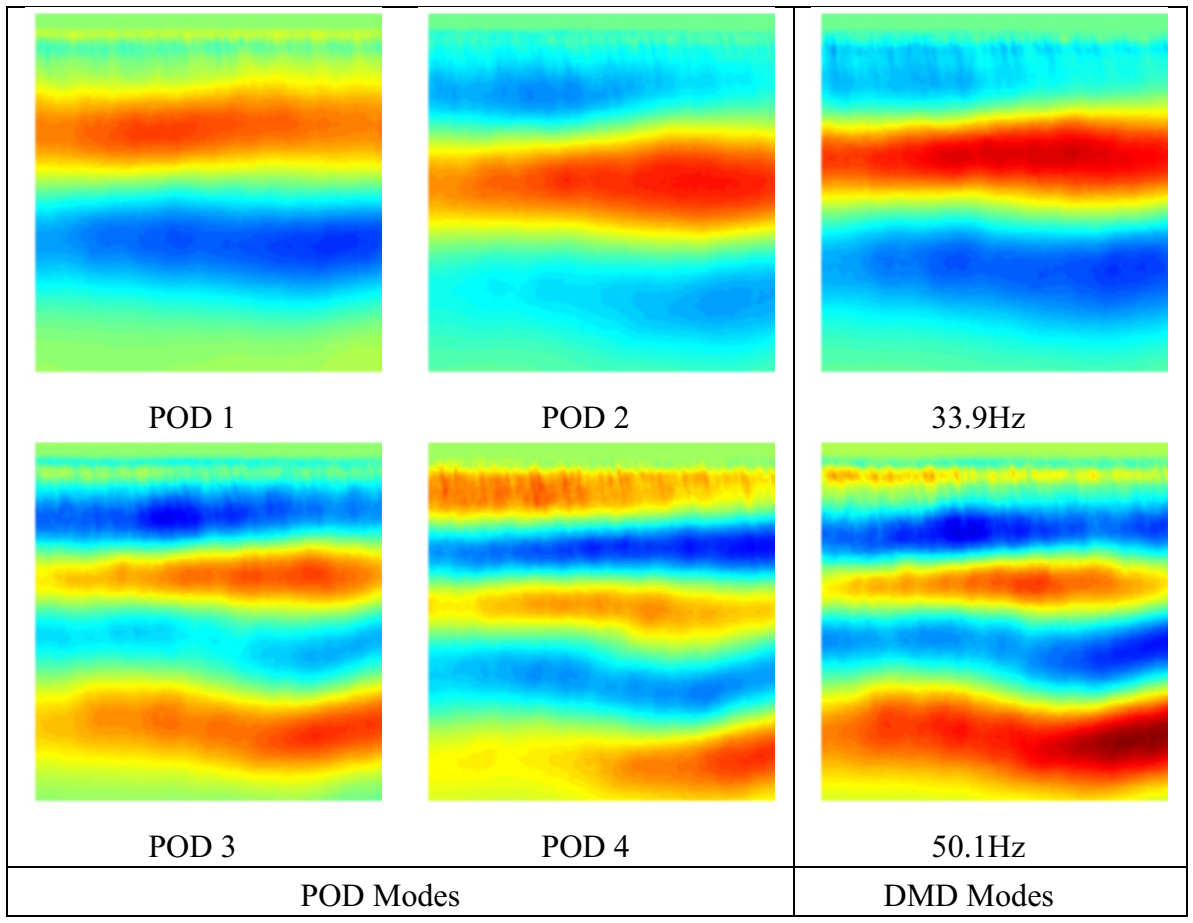


Fig. 22 Modes $(\sigma=1.5$, $\alpha=10^{\circ}$ )

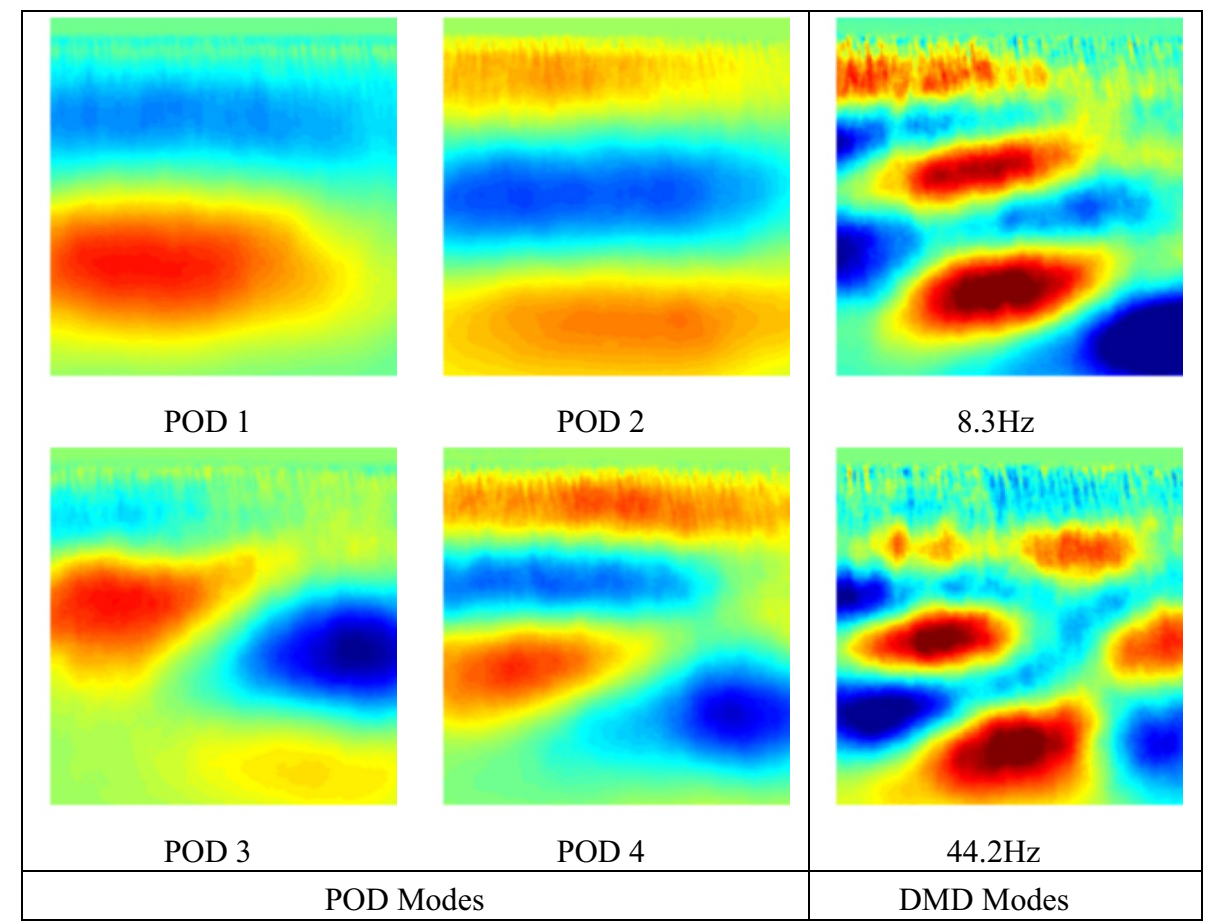

Fig. 23 Modes $(\sigma=1.25$, $\alpha=10^{\circ}$ )

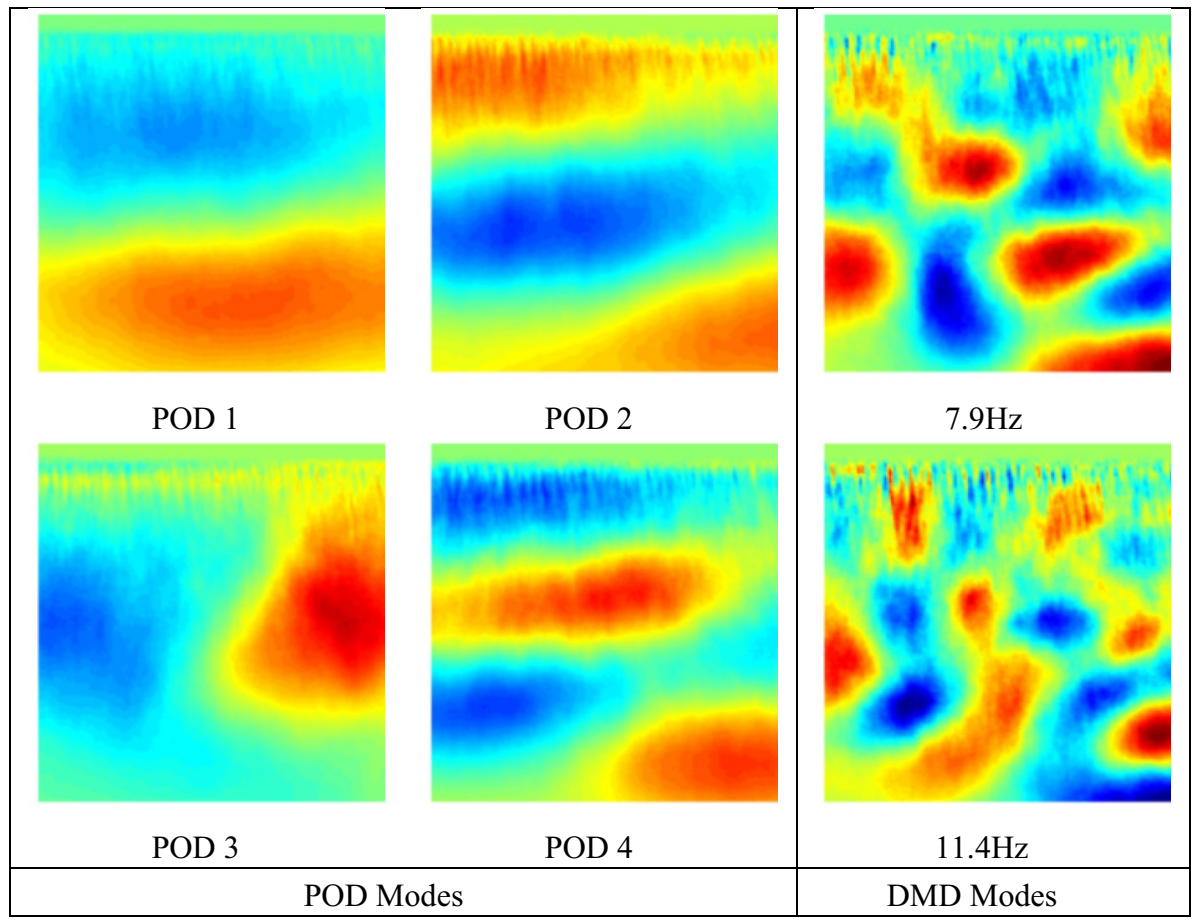

of sheet cavitation. However, for the third DMD mode $\sigma / 2 \alpha=5$, we see the 3D structures at low-frequency characteristics of the tearing instability caused by either the returning jet or by the shock wave. For $\sigma / 2 \alpha<4$, the DMD modes are associated with longitudinal 3D structures at low frequency. 


\section{Conclusions}

The joined POD and DMD methods applied to images allow the characterization of the spatio-temporal behavior of the sheet cavity which develops on the NACA0015 foil. These robust mathematical methods of signal postprocessing lead to the highlighting of the 3D effects due to the reentrant jet instabilities or due to propagating shock wave mechanisms at the origin of the shedding process of the cavitation cloud. Taking into account the parameter $\sigma / 2 \alpha$, the $2 \mathrm{D}$ and $3 \mathrm{D}$ spatial energetic trace of the vapor structures has been identified as well as frequency of each structure. Thus, the transition between the two mechanisms of instability corresponds to vapor structures associated with different spectral signatures. At the transition, the Strouhal number (based on chord length or cavity length) decreases drastically when the cavity length is in the range from 50 to $66 \%$ (Kawakami et al. 2008). The responsible mechanism is the nonlinear interaction between ripped off large- and small-scale vapor structures. As it was observed using POD analysis, the first modes characterize the two-dimensional structures at large scale (which are convected downstream by the longitudinal velocity) instead of the higher-order DMD modes show the presence of small-scale structures propagating orthogonally to the flow when $\sigma / 2 \alpha<4$. Thus, at the interface between liquid phase and vapor cavity, the propagation of the shock wave generates a shedding process at small scale with a low-frequency trace. Furthermore, the three-dimensional aspect is due to the tearing instability caused by the shock wave. Decaix and Goncalvès (2013) in a Venturi flow detect a cross instability at low frequency corresponding to a cross flow inside the recirculation area leading to a growth of the cavitation sheet in an opposite phase between the two Venturi sides.

The cross analysis of POD and DMD modes show that for periodical behavior of the flow, POD modes can be interpreted on a physical basis and be associated with DMD modes which add a frequency information not detected by the POD analysis. For aperiodic behavior, a 3D pattern has been previously observed and the physical interpretation using POD method only leads to that the first modes remain bidimensional. On the contrary, the different modes calculated using DMD method reveal the 3D behavior of the flow. In that case, the different frequencies that characterize the flow are detected with an associated spatial pattern unseen by POD analysis.

\section{References}

Arndt REA, Song CCS, Kjeldsen M, He J, Keller A (2000) Instability of partial cavitation: a numerical/experimental approach. In: Twenty-third symposium on naval hydrodynamics. National Academies Press, Val de Reuil, France
Chahine GL, Hsiao C-T (2000) Modeling 3D unsteady sheet cavities using a coupled URANS-BEM code. In: 23th symposium of naval hydrodynamics, Val de Reuil

Danlos A, Ravelet F, Coutier-delgosha O, Bakir F (2014) Cavitation regime detection through proper orthogonal decomposition: dynamics analysis of the sheet cavity on a grooved convergentdivergent nozzle. Int J Heat Fluid Flow 47:9-20

De Lange DF, de Bruin GJ (1998) Sheet cavitation and cloud cavitation, re-entrant jet and three-dimensionality. Appl Sci Res 58:91-114

Decaix J, Goncalvès E (2013) Investigation of three-dimensional effects on a cavitating venturi flow. Int J Heat Fluid Flow 44:576-595

Dular M, Bachert B, Stoffel B, Sirok B (2004) Relationship between cavitation structures and cavitation damage. Wear 257:1176-1184

Fujii A, Kawakami DT, Tsujimoto Y, Arndt REA (2007) Effect of hydrofoil shapes on partial and transitional cavity oscillations. J Fluids Eng 129:6-10. doi:10.1115/1.2734183

Furness RA, Hutton SP (1975) Experimental and theoretical studies of two-dimensional fixed-type cavities. J Fluids Eng 97(4):515521. doi:10.1115/1.3448098

Ganesh H, Makiharju SA, Ceccio SL (2014) Partial cavity shedding due to the propagation of shock waves in bubbly flows. In: Proceedings of the 30th symposium on naval hydrodynamics

Hekmati A, Ricot D, Druault P (2011) About the convergence of POD and EPOD modes computed from CFD simulation. Comput Fluids 50(1):60-71. doi:10.1016/j.compfluid.2011.06.018

Kawakami Y, Kato H, Yamaguchi H, Tagaya Y, Tanimura M (1997) Mechanism and control of cloud cavitation. J Fluids Eng 119:788-794

Kawakami DT, Fuji A, Tsujimoto Y, Arndt REA (2008) An assessment of the influence of environmental factors on cavitation instabilities. J Fluids Eng 130:1-8. doi:10.1115/1.2842146

Kjeldsen M, Arndt REA (2001) Joint time frequency analysis techniques: a study of transitional dynamics in sheet/cloud cavitation. In: CAV 2001: fourth international symposium on cavitation, June 20-23, 2001. California Institute of Technology, Pasadena, USA

Laberteaux KR, Ceccio SL (2001) Partial cavity flows. Part 1. Cavities forming on models without spanwise variation. J Fluid Mech 431:1-41

Le Q, Franc JP, Michel JM (1993) Partial cavities: pressure pulse distribution around cavity. J Fluids Eng 115:249-254

Lumley JL (1970) Stochastic tools in turbulence. Academic Press, Cambridge

Ma X, Karniadakis GE (2002) A low-dimensional model for simulating three-dimensional cylinder flow. J Fluid Mech 458:181-190. doi:10.1017/S0022112002007991

Noack B, Afanasiev K, Morzynsky M, Tadmor G, Thielle F (2003) A hierarchy of low dimensional models for the transient and posttransient cylinder wake. J Fluids Mech 497:335-363

Oberleithner K, Sieber M, Nayeri CN, Paschereit CO, Petz C, Hege H-C, Noack BR, Wygnanski I (2011) Three-dimensional coherent structures in a swirling jet undergoing vortex breakdown: stability analysis and empirical mode construction. J Fluid Mech 679:383-414. doi:10.1017/jfm.2011.141

Perrin R, Cid E, Cazin S, Sevrain A, Braza M (2007) Phase-averaged measurements of the turbulence properties in the near wake of a circular cylinder at high Reynolds number by 2C-PIV and 3C-PIV. Exp Fluids 42(1):93-109

Pham TM, Larrarte F, Fruman DH (1999) Investigation of unstable sheet cavitation and cloud cavitation mechanisms. J Fluids Eng 121:289-296

Prothin S, Djeridi H, Billard J-Y (2012) Traitement d'image utilisant la POD et la DMD pour l'étude du développement de la cavitation sur un NACA0015. In: Proceedings on des 13èmes journées de l'hydrodynamque 
Prothin S, Djeridi H, Billard J-Y (2013) Coherent and turbulent process analysis of the effects of a longitudinal vortex on boundary layer detachment on a NACA0015 foil. J Fluids Struct. doi:10.1016/j.jfluidstructs.2013.08.014

Rowley CW, Mezic I, Bagheri S, Schlatter P, Henningson DS (2009) Spectral analysis of nonlinear flows. J Fluid Mech 64:115-127

Schmid PJ (2010) Dynamic mode decomposition of numerical and experimental data. J Fluid Mech 656:5-28
Sirovich L (1987) Turbulence and the dynamics of coherent structures. Q Appl Math 45:561-590

Stutz B, Reboud L (1997) Experiments on unsteady cavitation. Exp Fluids 22:191-198

Van Oudheusden BW, Scarano F, VanHinsberg NP, Watt DW (2005) Phase-resolved characterization of vortex shedding in the near wake of a square-section cylinder at incidence. Exp Fluids 39(1):86-98 